\title{
Linear methods in band theory
}

\author{
Andersen, O. Krogh
}

Published in:

Physical Review B

Link to article, DOI:

10.1103/PhysRevB.12.3060

Publication date:

1975

Document Version

Publisher's PDF, also known as Version of record

Link back to DTU Orbit

Citation (APA):

Andersen, O. K. (1975). Linear methods in band theory. Physical Review B, 12(8), 3060-3083. https://doi.org/10.1103/PhysRevB.12.3060

\section{General rights}

Copyright and moral rights for the publications made accessible in the public portal are retained by the authors and/or other copyright owners and it is a condition of accessing publications that users recognise and abide by the legal requirements associated with these rights.

- Users may download and print one copy of any publication from the public portal for the purpose of private study or research.

- You may not further distribute the material or use it for any profit-making activity or commercial gain

- You may freely distribute the URL identifying the publication in the public portal

If you believe that this document breaches copyright please contact us providing details, and we will remove access to the work immediately and investigate your claim 


\title{
Linear methods in band theory*
}

\author{
O. Krogh Andersen \\ Department of Electrophysics, Technical University, Lyngby, Denmark
}

(Received 14 April 1975)

\begin{abstract}
Two approximate methods for solving the band-structure problem in an efficient and physically transparent way are presented and discussed in detail. The variational principle for the one-electron Hamiltonian is used in both schemes, and the trial functions are linear combinations of energy-independent augmented plane waves (APW) and muffin-tin orbitals (MTO), respectively. The secular equations are therefore eigenvalue equations, linear in energy. The trial functions are defined with respect to a muffin-tin (MT) potential and the energy bands depend on the potential in the spheres through potential parameters which describe the energy dependence of the logarithmic derivatives. Inside the spheres, the energy-independent APW is that linear combination of an exact solution, at the arbitrary but fixed energy $\boldsymbol{E}_{v}$, and its energy derivative which matches continuously and differentiably onto the plane-wave part in the interstitial region. The energies obtained with the linear-APW method for the MT potential have errors of order $\left(E-E_{v}\right)^{4}$. Similarly, the energy-independent MTO is that linear combination which matches onto that solution of the Laplace equation in the interstitial region which is regular at infinity. The energies obtained with the linear-MTO method have additional errors of order $\left(E-V_{\mathrm{mtz}}\right)^{2}$, arising from the interstitial region where the potential is $V_{\mathrm{mtz}}$. The linear-APW (LAPW) method combines desirable features of the APW and OPW methods; it can treat $d$ bands, the energy dependence of its pseudopotential is linear and, owing to the smoothness of the energy-independent APW at the spheres, non-MT contributions to the potential are included principally through their Fourier components. The linear-MTO (LMTO) method is particularly suited for closely packed structures and it combines desirable features of Korringa-Kohn-Rostoker, linear-combination-of-atomic-orbitals, and cellular methods; the secular matrix is linear in energy, the overlap integrals factorize as potential parameters and structure constants, the latter are canonical in the sense that they neither depend on the energy nor the cell volume and they specify the boundary conditions on a single MT or atomic sphere in the most convenient way. This method is very well suited for self-consistent calculations. The empty-lattice test is applied to the linear-MTO method and the free-electron energy bands are accurately reproduced. Finally, it is shown how relativistic effects may be included in both the LAPW and LMTO methods.
\end{abstract}

\section{INTRODUCTION}

During the past fifteen years the techniques for solving the band-structure problem have reached the point at which, with the aid of large computers, an accurate solution may be obtained. Although the one-electron problem may, in principle, be solved ${ }^{\circ}$ with arbitrary accuracy for a given periodic potential, particularly if it has the muffin-tin (MT) form appropriate for closely packed elements, in practice a comparison of different calculations for the same MT potential reveals computational discrepancies in the energy eigenvalues as great as $0.01 \mathrm{Ry}$, provided that they lie less than about 1 Ry above the bottom of the conduction band. For open structures and higher energies the accuracy is lower.

The problem of constructing one-electron potentials has been solved to the extent that, for nonmagnetic closely packed elements, we can, by a judicious choice of configuration and the exchangecorrelation contribution, arrive at potentials which reproduce the experimental Fermi surfaces with an accuracy also equivalent to about $0.01 \mathrm{Ry}$. This is the accuracy, for example, of transition-metal potentials, as discussed in more detail in the companion paper by Jepsen, Mackintosh, and Andersen, ${ }^{1}$ hereafter referred to as II. Recent develop- ments in the theory of local exchange correlation potentials $\mathrm{s}^{2}$ and attempts to use them $\mathrm{m}^{3,4}$ have raised the hope that, if we can solve the band-structure problem self-consistently, we may, in an unambiguous and realistic way, compute ground-state properties such as cohesive energies, interatomic forces, charge transfer, and magnetic moments, and also excitation spectra described by the oneelectron Green's function.

However, the augmented plane wave $\mathrm{F}^{5}$ (APW) and the Korringa-Kohn-Rostoker ${ }^{6}$ (KKR) methods, which have allowed the solution of the band-structure problem for a wide class of materials, require a computational effort which, despite recent attempts to improve the efficiency, is barely feasible in truly self-consistent calculations. This is particularly so in the calculation of ground-state properties of compounds and magnetic crystals, where self-consistency is imperative, and in the calculation of excitation spectra, where matrix elements are needed. Moreover, the APW and KKR methods still have the characteristics of numerical techniques, well suited for accurate calculations on particular crystals, but not so far amenable to obvious simplifications, in contrast to the way in which the cellular, the orthogonalized-plane-wave (OPW) and the linearcombination-of-atomic-orbitals (LCAO) methods 
have lead to, respectively, the spherical, the pseudopotential, and the tight-binding approximations.

A substantial effort to derive from the KKR formalism a simple, yet general, scheme based on the concepts of pseudopotentials and $d$-wave resonances was initiated by Ziman, Hubbard, Heine, and others, ${ }^{7}$ and it has reached its most sophisticated development in the work of Pettifor. ${ }^{8}$ His hybrid nearly-free-electron tight-binding scheme is computationally fast and can yield accurate energies, but it cannot treat broad $d$ bands as, for instance, those of the alkaline-earth metals or the early transition metals. Furthermore, the phase-shift parameters are nonunique. ${ }^{9}$ We feel that, in these attempts, the KKR method has been changed too drastically and, since the band structures achieved from the APW and KKR methods exhibit gradual changes as one proceeds through the Periodic Table of elemental crystalline solids, it is difficult to justify treating $s, p, d$, and (in the case of the light actinides) $f$ bands in radically different ways. In this paper we shall therefore present APW- and KKR-like formalisms which have the virtues lacking in the original formulations, simply by using energy-independent APWs of a type previously mentioned by Marcus ${ }^{10}$ and energy-independent muffin-tin orbitals (MTOs) of a type suggested by the author. ${ }^{11,12}$

The obvious computational advantage of using the variational principle for Schrödinger's equation with energy-independent basis functions is that the secular equations become linear in energy, that is, they reduce to the eigenvalue equations

$$
\sum_{L}\left(H_{L^{\prime} L}^{\overrightarrow{\mathbf{k}}}-E_{j}^{\overrightarrow{\mathbf{k}}} O_{L^{\prime} L}^{\overrightarrow{\mathbf{k}}}\right) A_{L j}^{\overrightarrow{\mathbf{k}}}=0
$$

from which all eigenvalues $E_{j}^{\overrightarrow{\mathrm{k}}}$ and eigenvectors $A_{L j}^{\overrightarrow{\mathrm{k}}}$ of a given Bloch vector $\overrightarrow{\mathrm{k}}$ can be found simultaneously by diagonalization procedures, ${ }^{13}$ and the wave functions are in a form convenient for further use, e.g., they are strictly orthonormal. In order to employ Schrödinger's equation, instead of the equivalent integral equation, we shall use the linear-. combination-of-MTO (LCMTO) formalism ${ }^{12,14}$ rather than the equivalent KKR formalism.

Both the energy-independent and the conventional APWs and MTOs are defined with respect to a MT potential but, while the conventional basis functions are constructed inside the MT spheres from the solutions $\phi_{l}(E, r)$ of the radial Schrödinger equations at the energy in question, the energy-independent basis functions are constructed from linear combinations of the functions $\phi_{l}\left(E_{\nu}, r\right)$ and their energy derivatives $\dot{\phi}_{l}\left(E_{\nu}, r\right)$ at the arbitrary, but fixed, energy $E_{\nu}$. This has the consequence that the linear-APW and -MTO methods are no longer exact for the MT potential but the errors of the energies and wave functions are, respectively, only of fourth and second order in the difference $E_{j}^{\overrightarrow{\mathrm{k}}}-E_{\nu}$.
In practice, the fourth-order errors of the energies amount to less than $1 \%$ of the bandwidth over its entire range, which for wide $s$ and $p$ bands is of order $1 \mathrm{Ry}$. Moreover, in the linear-MTO method it is easy to obtain wave functions correct to third order. As in the original formalisms, the potential in the spheres only enters through the logarithmic derivative functions $S \phi_{l}^{\prime}(E, S) / \phi_{l}(E, S)$ at the sphere or rather, through the parameters of these functions of energy, and our potential parameters are thus generalizations of the parameters used by Bardeen, Silverman, and Brooks ${ }^{15}$ for describing the position, mass, and higher-order terms of the bottom of a simple conduction band.

In the interstitial region, outside the MT spheres, the energy-independent APWs are plane waves whereas the energy-independent MTOs have a more: restricted form; they are solutions of the Laplace equation, i.e., they have zero kinetic energy. In contrast to the KKR structure constants, those of the linear-MTO (LMTO) method are therefore $c a$ nonical in the sense that they neither depend on the energy nor on the atomic volume, and they can be tabulated once and for all for a given structure throughout the corresponding Brillouin zone. In the approximation that the overlap integrals in the interstitial region are substituted by integrals in the shell between the MT and atomic Wigner-Seitz spheres, these canonical structure constants $S_{l^{\prime} m^{\prime} ; l m}^{\vec{k}}$ specify the anisotropic and $\vec{k}$-dependent boundary condition on a single atomic sphere. The eigenvalues of the diagonal blocks $S_{l m^{\prime} ; l m}^{\vec{k}}$ define canonical $l$ bands from which the unhybridized energy-band structure may be derived by scaling with the abovementioned potential parameters, provided that they are defined at the atomic sphere. When the integrals in the interstitial region are taken into account properly, the choice of vanishing kinetic energy for the MTOs in this region leads to errors in the energies of order $\left(E_{j}^{\overrightarrow{\mathrm{k}}}-V_{\mathrm{mtz}}\right)^{2}$, where $V_{\mathrm{mtz}}$ is the potential in the interstitial region. For closely packed structures, and for energies no more than 1 Ry above $V_{\mathrm{mtz}}$, these errors rarely exceed $0.01 \mathrm{Ry}$.

The energy-independent APWs form a complete set of functions in the interstitial region and, since, contrary to the conventional APWs, they are differentiable at the MT spheres, they are particularly suited for open structures where nonMT contributions to the potential are important. In fact, the linear-APW (LAPW) method is quite similar to, and has all the advantages of, the OPW method often used for semiconductors; but, in addition, it treats $d$ bands as readily as the conventional APW method, and there is no need for explicit core state orthogonalizations because $\phi_{l}\left(E_{\nu}, r\right)$ and $\dot{\phi}_{l}\left(E_{\nu}, r\right)$ are exactly orthogonal to the core states of the MT well. 
The convergence of the LAPW and LMTO methods are essentially the same as for the conventional nonlinear APW and KKR methods, which means that 4-60 energy-independent APWs and 4-16 energyindependent MTOs are needed per atom in the primitive cell; the smaller numbers pertain to the simplest metals while the larger numbers pertain to open structures and situations in which the bands have a significant $f$ character. The computational speed of our methods is largely determined by the diagonalization techniques for the following reasons: the radial Schrödinger equations need only be solved at a few energies; the Hamiltonian and overlap matrices are rather simple, particularly those of the LAPW method; and the structure constants of the LMTO method can be computed and stored once and for all. Owing to the smaller matrix size in the latter method, it is generally to be preferred and with our ${ }^{16}$ present nonrelativistic LMTO routines for fcc and bcc crystals, we obtain all nine eigenvalues per $\overrightarrow{\mathrm{k}}$ point in less than $0.1 \mathrm{sec}$ with an IBM $360 / 75$ computer. The computing times for our relativistic routines for hcp crystals will be reported in II. If we neglect the $s p d$ hybridization and use the above-mentioned atomic-sphere approximation (ASA), which is not equivalent with the spherical approximation to the cellular method, there are no diagonalizations to perform, and a self-consistency cycle, therefore, merely consists of solving the radial Schrödinger equations, scaling the canonical state densities by the potential parameters, finding the Fermi energy, . constructing the spherically symmetrized charge density, and solving the radial Poisson equation. This procedure may be accomplished in a few seconds, and Madsen et al. ${ }^{4}$ have used it to study the behavior of the magnetic moment of $\mathrm{Fe}$ as function of atomic volume and crystal structure. At points of high symmetry in the Brillouin zone, where hybridization is weak or absent, the energies may be computed by hand from tables of the canonical bands and potential parameters. ${ }^{16}$

For reasons of length this paper will be limited to a presentation of the LAPW and LMTO formalisms and a discussion of their accuracy. Unpublished accounts of these formalisms, together with applications to transition metals, may be found in Ref. 11, while the simple concepts of the LMTO method, i.e., the canonical bands and the potential parameters, were briefly communicated in Ref. 17. A more general linear-MTO formalism for molecules was presented by Andersen and Woolley. ${ }^{12}$ In II may be found a detailed study of the electronic structures of the hcp transition metals using the LMTO method. Koelling and Arbman ${ }^{18}$ have investigated the computational aspects of the LAPW method, while the self-consistent-LMTO method is currently being applied to the compounds $\mathrm{Pd}_{3} \mathrm{Fe}$ and $\mathrm{V}_{3} \mathrm{Ga}$ by Jepsen ${ }^{19}$ and by Jarlborg and Arbman, ${ }^{20}$ respectively.

The organization of the present paper is as follows. In Sec. II we consider a single MT or atomic sphere and derive the $\phi-\dot{\phi}$ formalism common to the two linear methods. In Sec. II A we present the basic formalism, and in Sec. II B we make a specific choice of potential parameters, which will prove useful at later stages, and give relations between them. In Sec. II $\mathrm{C}$ we discuss the accuracy and show how the potential parameters are related to the coefficients in a Laurent series for the exact logarithmic derivative function, and in Sec. II D we give as an example, and for later use, expressions for the potential parameters of free electrons. The LAPW method is described in Sec. III. The basic formalism may be found in Sec. III A, while the accuracy and convergence properties are discussed in Sec. III B, non-MT contributions are introduced in Sec. III C, and a comparison with other APW formalisms is made in Sec. III D. The LMTO method is discussed in Sec. IV. We first define the energyindependent MTO in Sec. IVA, and give its onecenter expansion. In Sec. IV B we write down the Hamiltonian and overlap matrices, first in the ASA, and then include a combined correction for the neglect of the corners of the atomic polyhedron and the higher partial waves. In Sec. IV C we discuss the accuracy and show, by comparison with the KKR formalism in the ASA, how the one-center expansion of the wave function, correct to third order in $E_{j}^{\overrightarrow{\mathrm{k}}}-E_{\nu}$, may be derived from the LMTO eigenvectors. In Sec. IV D we give expressions for the canonical structure constants, define the canonical bands, and discuss their properties, in particular their first and second moments. The physical significance of the potential parameters becomes clear in Sec. IVE, where we show how they determine the positions, widths, and distortions of the unhybridized energy bands relative to those of the canonical bands. As an example, we consider the free-electron bands in the hcp structure. In Sec. IV $\mathrm{F}$ the LMTO formalism is extended to the case of several atoms per primitive cell, and we give the expression for the spherically symmetrized electron density in the ASA. Relativistic effects are included in Sec. V, where we use the Schrödinger rather than the Pauli representation because the spin-orbit splittings encountered in all solids, except perhaps the actinides, are at least one order of magnitude smaller than the relevant bandwidths. In Sec. VA we show how the potential parameters, with the addition of the spin-orbit coupling parameter and a rormalization parameter, determining the dependence of the relativistic effects on the position within the band, may be derived from the solutions of the radial Dirac equations. The LAPW and LMTO spin-orbit coupling matrices are then given 
in Secs. VB and VC, respectively.

This paper is rather lengthy but has the virtue of communicating in a comprehensive manner the formalism of the linear methods. The essence of the LAPW method may be found in Secs. II A, III A, and VB, while a concise description of the LMTO method is given in Sec. II of the companion paper II.

\section{LINEAR METHOD FOR SINGLE SPHERE}

In this section, we shall present an approximation, valid in the neighborhood of some arbitrary energy $E_{\nu}$, to the eigenvalues of a single atomic or muffintin sphere of radius $S$, and with the spherically symmetric Hamiltonian $-\nabla^{2}+v(r)$ (in atomic rydberg units).

\section{A. Basic formalism}

As radial basis functions, for each value of $l$, we use the solution

$$
\phi_{\nu l}(r) \equiv \phi_{l}\left(E_{\nu}, r\right)
$$

of the radial Schrödinger equation for $v(r)$ and its energy derivative

$$
\left.\dot{\phi}_{\nu l}(r) \equiv \frac{\partial \phi_{l}(E, r)}{\partial E}\right|_{E_{\nu}}
$$

In the following, we shall often omit the subscript $l$. The normalization is specified through

$$
\left\langle\phi^{2}(E)\right\rangle=\int_{0}^{S} \phi^{2}(E, r) r^{2} d r \equiv 1 .
$$

Consequently, $\phi_{\nu}(r)$ and $\dot{\phi}_{\nu}(r)$ are orthogonal, and it may be shown that they are both orthogonal to the core states. The radial logarithmic derivatives at the sphere boundary

$$
D_{\nu} \equiv S \phi_{\nu}^{\prime}(S) / \phi_{\nu}(S)
$$

and

$$
D_{\dot{\nu}} \equiv S \dot{\phi}_{\nu}^{\prime}(S) / \dot{\phi}_{\nu}(S)
$$

are always different, as may be seen from (2.9), and a trial function of arbitrary logarithmic derivative $D$ is therefore the linear combination

$$
\Phi(D, r)=\phi_{\nu}(r)+\omega(D) \dot{\phi}_{\nu}(r),
$$

where, in addition to $l$, we have omitted a subscript $\nu$ on $\Phi$ and $\omega$. In Fig. 1 we show $\phi_{4 d}, \dot{\phi}_{4 d}$, and $\ddot{\phi}_{4 d}$ for yttrium.

In the basis of

$$
\Phi_{l m}(D, \overrightarrow{\mathrm{r}}) \equiv i^{\imath} Y_{\imath m}(\hat{r}) \Phi_{\imath}(D, r),
$$

where $Y_{l m}$ are the spherical harmonics with the phase of Ref. 21, the Hamiltonian matrix elements in the sphere are given by

$$
\left\langle\Phi_{l^{\prime} m^{\prime}}\left(D^{\prime}\right)\left|H-E_{\nu}\right| \Phi_{l m}(D)\right\rangle=\delta_{l^{\prime}{ }_{l}} \delta_{m^{\prime} m} \omega_{l}(D) \text {, }
$$

as obtained by differentiation of Schrödinger's equation $(H-E) \phi(E, \overrightarrow{\mathrm{r}})=0$ with respect to energy,

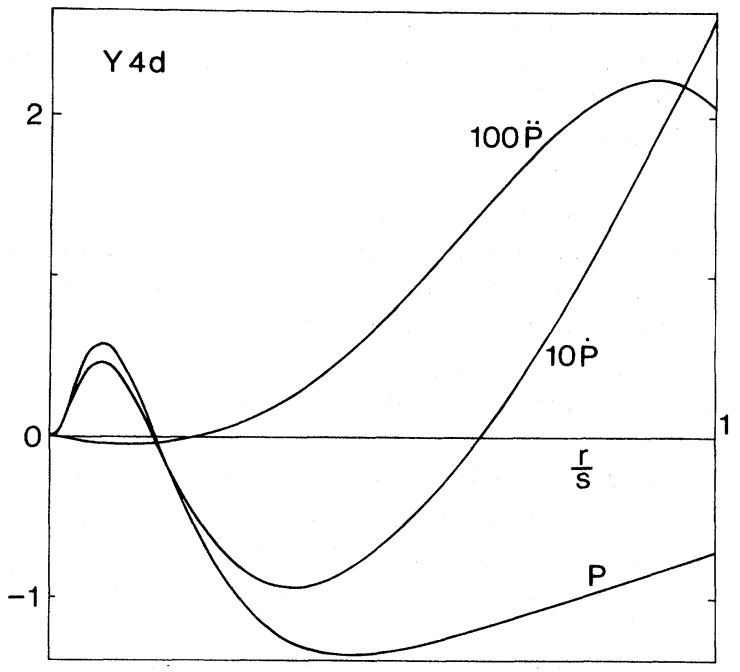

FIG. 1. $4 d$ energy derivative functions with $D_{y}=-3$ for yttrium. $P \equiv S^{1 / 2} \gamma \phi_{\nu}, \dot{P} \equiv S^{-2} \partial P / \partial E$, and $\ddot{P} \equiv S^{-4} \partial^{2} P / \partial E^{2}$.

that is,

$$
\left(H-E_{\nu}\right) \dot{\phi}_{\nu}(\overrightarrow{\mathrm{r}})=\phi_{\nu}(\overrightarrow{\mathrm{r}}) .
$$

The normalization integrals are

$$
\begin{aligned}
\left\langle\Phi_{l^{\prime} m^{\prime}}\left(D^{\prime}\right) \mid \Phi_{l m}(D)\right\rangle= & \delta_{l^{\prime}{ }_{l} \delta_{m}{ }^{\prime} m} \\
& \times\left[1+\left\langle\dot{\phi}_{\nu l}^{2}\right\rangle \omega_{l}\left(D^{\prime}\right) \omega_{l}(D)\right],
\end{aligned}
$$

and this expression will, together with (2.4), form the basis for our derivation of the linear-APW and -MTO methods in Secs. III and IV.

\section{B. Potential parameters}

When in the following sections we consider the band-structure problem, we shall join together the trial functions from all spheres of the solid. The influence of each sphere on the energy spectrum will then be given as a set of potential parameters, which are the parameters $\left\langle\dot{\phi}_{\nu l}^{2}\right\rangle$, plus the parameters of the energy functions $\omega_{l}(D)$ and the amplitude functions $\Phi_{l}(D, S) \equiv \Phi_{l}(D)$. From (2.2)

$$
\omega(D)=-\frac{\phi_{\nu}}{\dot{\phi}_{\nu}} \frac{D-D_{\nu}}{D-D_{\nu}}
$$

and

$$
\Phi(D)=\phi_{\nu} \frac{D_{\nu}-D_{\dot{\nu}}}{D-D_{\dot{\nu}}},
$$

as specified by the logarithmic derivatives $D_{\nu}$ and $D_{\dot{\nu}}$, and the amplitudes $\phi_{\nu}$ and $\dot{\phi}_{\nu}$, at the sphere. For the latter we have adopted the notation $\phi_{\nu} \equiv \phi_{\nu}(S)$ and $\dot{\phi}_{\nu} \equiv \dot{\phi}_{\nu}(S)$. Of these parameters, only three are independent since from (2.1), from (2.5), and by use of Green's second identity,

$$
1=\left\langle\phi_{\nu}^{2}\right\rangle=\left\langle\phi_{\nu}\left|H-E_{\nu}\right| \dot{\phi}_{\nu}\right\rangle
$$




$$
=\left(D_{\nu}-D_{\dot{\nu}}\right) S \phi_{\nu} \dot{\phi}_{\nu}
$$

The functions $\omega(D)$ and $\Phi(D)$ are thus related by

$$
S \Phi^{2}(D)=-\frac{d \omega}{d D} .
$$

If, instead of the amplitudes, we use $S \phi_{\nu}^{2}$ and $S \phi_{\nu} \dot{\phi}_{\nu}$, the sphere radius will not be a separate parameter. For each sphere and for each value of the radial and angular quantum numbers $\nu$ and $l$, there are therefore four independent potential parameters, e.g. , $D_{\nu}, S \phi_{\nu}^{2}, S \phi_{\nu} \dot{\phi}_{\nu}$, and $\left\langle\dot{\phi}_{\nu}^{2}\right\rangle$.

The values of the first three parameters depend strongly on the choice of $E_{\nu}$, and it will be more convenient to use $\omega\left(D_{1}\right), S \Phi^{2}\left(D_{1}\right)$, and $\Phi\left(D_{1}\right) / \Phi\left(D_{2}\right)$, which refer to specific boundary conditions $D_{1}$ and $D_{2}$. The choices $D_{1}=-l-1$ and $D_{2}=l$ will prove useful, and with them we obtain alternatively to (2.2), (2.7), and (2.8), respectively,

$$
\begin{aligned}
& \Phi(D, r)= \frac{\omega(D)-\omega(-l-1)}{\omega(l)-\omega(-l-1)} \Phi(l, r) \\
& \quad+\frac{\omega(D)-\omega(l)}{\omega(-l-1)-\omega(l)} \Phi(-l-1, r), \\
& \frac{\omega(D)-\omega(-l-1)}{\omega(D)-\omega(l)}=\frac{\Phi(-l-1)}{\Phi(l)} \frac{D+l+1}{D-l},
\end{aligned}
$$

and

$$
\Phi(D)=\frac{(2 l+1) \Phi(-l-1) \Phi(l)}{(D+l+1) \Phi(-l-1)-(D-l) \Phi(l)} .
$$

The relation implied by (2.9) is

$$
S \Phi\left(D_{1}\right) \Phi\left(D_{2}\right)=-\left[\omega\left(D_{2}\right)-\omega\left(D_{1}\right)\right] /\left(D_{2}-D_{1}\right),
$$

and with our choices of $D_{1}$ and $D_{2}$

$$
(2 l+1) S \Phi(-l-1) \Phi(l)=\omega(-l-1)-\omega(l) .
$$

In the first place, the $(\omega, \Phi)$ parameters are obtained from $D_{\nu}, S \phi_{\nu}^{2}$, and $S \phi_{\nu} \dot{\phi}_{\nu}$ using (2.7)-(2.9). Subsequently, the latter parameters may be rederived from tabulated values of the former; $D_{\nu}$ is obtained from (2.12) when setting $D=D_{\nu}$ and $\omega(D)$ $=0$, the value of $S \phi_{\nu}^{2}$ follows from (2.11) evaluated at the sphere, and the value of $S \phi_{\nu} \dot{\phi}_{\nu}$ may finally be obtained from (2.7) and (2.9) when setting $D=-l-1$.

\section{Accuracy}

We now compare the above-mentioned linear approach leading to (2.4) and (2.6) with the exact one followed in the APW and KKR methods for the single sphere. In these methods, all information about the sphere, for a particular value of $l$, is carried by the logarithmic derivative function

$$
D(E) \equiv S \phi^{\prime}(E, S) / \phi(E, S)
$$

obtained by integrations of the radial Schrödinger equation and regarding the energy as the independent variable. This function may be expressed in terms of the eigenvalues $E_{n}(n=0,1, \ldots, \nu, \ldots)$, specified by the boundary condition $D\left(E_{n}\right)=D_{\nu}$, and the corresponding partial probability densities $\phi_{n}^{2}$ at the sphere as

$$
\frac{1}{D(E)-D_{\nu}}=-\sum_{n} \frac{S \phi_{n}^{2}}{E-E_{n}} .
$$

This representation of the logarithmic derivative function, which is implicit in the work of Saffren ${ }^{22}$ and is derived here, shows that $D(E)$ is an ever decreasing, cot-like function. In the linear approach, we estimate the $\nu$ th branch of the inverse function of $D(E)$, that is, the eigenvalue defined by the quantum numbers $\nu l$ plus the boundary condition $D$, by use of the variational principle for the Hamiltonian and with the trial function $\Phi(D, \overrightarrow{\mathrm{r}})$. From (2.4) and (2.6), this is

$$
E(D)=\frac{\omega(D)}{1+\left\langle\phi_{\nu}^{2}\right\rangle \omega^{2}(D)}+E_{\nu} .
$$

It is intuitively obvious that the difference between $\Phi(D(E), r)$ and the correct wave function $\phi(E, r)$ is of order $\left(E-E_{\nu}\right)^{2}$, whereby the error of the variational estimate $(2.17)$ is of order $\left(E-E_{\nu}\right)^{4}$. For the wave functions, it is then consistent to use the Taylor series to third order in energy of $\phi(E(D), r)$ instead of the first-order estimate $\Phi(D, r)$. We now prove (2.16), consider the accuracy of (2.17) in detail, and give relations between the potential parameters, the logarithmic derivative function, and the second and third energy derivative functions.

The formal expansion

$$
\sum_{n}\left\langle\phi(E) \mid \phi_{n}\right\rangle \phi_{n}(r)
$$

of $\phi(E, r)$ converges at the sphere to $\phi(E, S)$, although its logarithmic derivative is $D_{\nu}$ rather than $D(E)$. The expansion coefficients may be obtained from

$$
\begin{aligned}
0 & =-\left\langle\phi_{n}|H-E| \phi(E)\right\rangle \\
& =\left(E-E_{n}\right)\left\langle\phi(E) \mid \phi_{n}\right\rangle+\left[D(E)-D_{\nu}\right] S \phi(E) \phi_{n},
\end{aligned}
$$

whereby (2.16) is proved. From this representation of $D(E)$ we realize that its $\nu$ th "period" may be described by the Laurent series

$\frac{1}{D(E)-D_{\nu}}=-\frac{S \phi_{\nu}^{2}}{\epsilon}+\sum_{n}^{\prime} \frac{S \phi_{n}^{2}}{E_{n \nu}}+\epsilon \sum_{n}^{\prime} \frac{S \phi_{n}^{2}}{E_{n \nu}^{2}}+o(\epsilon)$,

where $\epsilon \equiv E-E_{\nu}$ and $E_{n \nu} \equiv E_{n}-E_{\nu}$. For the second term we find

$$
\sum_{n}^{\prime} S \phi_{n}^{2} E_{n \nu}^{-1}=-S \phi_{\nu} \dot{\phi}_{\nu}=\left(D_{\nu}-D_{\nu}\right)^{-1}
$$


by substituting the Taylor series

$$
\phi(E, r)=\phi_{\nu}(r)+\epsilon \dot{\phi}_{\nu}(r)+o(\epsilon)
$$

into (2.18). By use of (2.7) and (2.9) we may then write (2.19) as

$$
\frac{1}{\omega(D(E))}=\frac{1}{\epsilon}-\frac{\epsilon}{S \phi_{\nu}^{2}} \sum_{n}^{\prime} \frac{S \phi_{n}^{2}}{E_{n \nu}^{2}}-o(\epsilon),
$$

which proves that $\omega(D(E))+E_{\nu}$ is the correct energy to order $\epsilon^{2}$. The error of the trial function $\Phi(D(E), r)$ is then, by comparison of (2.2) with (2.21), of order $\epsilon^{2}$, and the error of the variational estimate is of order $\epsilon^{4}$, that is,

$$
E(D(E))=E+o\left(\epsilon^{3}\right) .
$$

Also, the fourth logarithmic derivative parameter $\sum_{n}^{\prime} S \phi_{n}^{2} E_{n \nu}^{-2}$ in (2.19) may be expressed in terms of previously defined potential parameters. From (2.17) and (2.23)

$$
\epsilon^{-1}=\omega^{-1}(D(E))+\left\langle\dot{\phi}_{\nu}^{2}\right\rangle \omega(D(E))+o(\epsilon)
$$

and, by comparison with (2.22),

$$
\begin{aligned}
\sum_{n}^{\prime} S \phi_{n}^{2} E_{n \nu}^{-2} & =S \phi_{\nu}^{2}\left\langle\dot{\phi}_{\nu}^{2}\right\rangle \\
& =-S \phi_{\nu}^{2}\left\langle\phi_{\nu} \mid \ddot{\phi}_{\nu}\right\rangle=-\frac{1}{3} S \phi_{\nu} \ddot{\phi}_{\nu} \\
& =-\frac{1}{3}\left(D_{\nu}-D_{\nu} \cdot\right) S \phi_{\nu}^{2} S \phi_{\nu} \ddot{\phi}_{\nu} .
\end{aligned}
$$

The three latter expressions may be derived from (2.18) with $n=\nu$ by substituting the Taylor series to third order for $\phi(E, r)$ and $\phi^{\prime}(E)$ and making use of the normalization condition (2.1). It then further appears that $D_{\ddot{\nu}}=D_{\nu}$.

The difference between the variational estimate and that of the Laurent series, truncated after the first three terms, is of higher order than the error of either estimate. This is so because $\omega(D(E))+E_{\nu}$ equals the energy to second order, rather than to first order, whereby the functions $o(\epsilon)$ in (2.22) and (2.24) are identical to second order. The leading term in the error of the variational estimate (2.23) is then

$$
\epsilon^{4} \sum_{n}^{\prime}\left(\frac{\phi_{n}}{\phi_{\nu}}\right)^{2} E_{n \nu}^{-3}
$$

which, as expected, is positive when $\phi_{\nu}(r)$ is the first state above the core states for which $\phi_{n} \simeq 0$. The width of a conduction band in a closely packed solid is of order $\frac{1}{2} E_{(\nu+1) \nu}$ and the amplitudes $\left|\phi_{\nu}\right|$ and $\left|\phi_{\nu+1}\right|$ are nearly equal. If we choose $E_{\nu}$ at the middle of this band, the error at the band edges is seen to be around $\frac{1}{128}$ of the bandwidth. Hence, the linear approximation is acceptable over the entire bandwidth.

The variational estimate (2.17) is confined to the neighborhood of $E_{\nu}$, regardless of whether $\Phi_{\nu}(D, r)$ is orthogonal to the lower-lying states of the same logarithmic derivative or not, specifically,

$$
\left|E(D)-E_{\nu}\right| \leq \frac{1}{2}\left\langle\dot{\phi}_{\nu}^{2}\right\rangle^{-1 / 2}
$$

Considering again a conduction band, and making use of (2.25), we realize that the range set by (2.26) extends approximately $\frac{1}{2} E_{(\nu+1) \nu}$ on either side of $E_{\nu}$, that is, twice as far as the band edges. Hence, the $\nu$ th branch of the inverse function of $D(E)$ is almost spanned by the variational estimate, but for logarithmic derivatives in the range around $D_{\dot{v}}$, specified by

$$
\left|\left(D-D_{\nu}\right)^{-1}-\left(D_{\nu}-D_{\nu}\right)^{-1}\right| \leq S \phi_{\nu}^{2}\left\langle\dot{\phi}_{\nu}^{2}\right\rangle^{1 / 2},
$$

the estimate $E(D)$ is quite erroneous. This is clearly seen in Fig. 2 where we have shown, for free $s$ electrons, the true logarithmic derivative function, $D(E)=S(\sqrt{E}) \cot [S(\sqrt{E})]-1$, together with the three-term Laurent estimate, the variational estimate, and $\omega(D(E))$. We have used $S^{2} E_{\nu}=\frac{1}{4} \pi^{2}$, and the values of the potential parameters are derived in Sec. IID. As discussed in Sec. IV D, the boundary conditions at an atomic sphere of a closely packed solid only permit a band of $l$ character in the range of energies where $D_{l}(E)$ is negative, and only in this range is an accurate description of the logarithmic derivative function needed. If, therefore, a linear method is employed, and $E_{\nu}$ is such that, for one of the $l$ 's included, (2.27) defines a range of negative logarithmic derivatives, one part of the $l$ band will appear far below $E_{\nu}$ while the remaining part will appear far above $E_{\nu}$, and the two parts will be connected by steep unphysical bands. Such a situation might arise in a computation focusing on the $4 s-4 p$ bands of $\mathrm{Cu}$, with $E_{\nu}$ lying between the $3 d$ and $4 d$ bands so that $D_{\nu d}$ is positive. If $D_{\dot{\nu} d}$ turns out to be negative, a linear method which can and must yield five continuous $d$ bands does not know whether to pick the $3 d$ or $4 d$ bands; it therefore picks one part of each. The disturbing unphysical bands may, however be swept out of the range of interest simply by setting $\left\langle\dot{\phi}_{\nu l}^{2}\right\rangle=0$ for the $l$ in question, whereby $E_{l}(D)$ is substituted by the monotonic function $\omega_{l}(D)$.

\section{Free-electron parameters}

The potential parameters for free electrons were used in Fig. 2 and will be referred to in the following. We shall therefore give expressions for them. The radial wave functions of kinetic energy $K^{2}$ are the normalized spherical Bessel functions

$$
\tilde{\phi}_{l}\left(K^{2}, r\right)=j_{l}(K r)\left\langle j_{l}^{2}(K r)\right\rangle^{-1 / 2}
$$

and the logarithmic derivative functions are

$$
\tilde{D}_{l}\left(K^{2}\right)=x j_{l}^{\prime}(x) / j_{l}(x),
$$

where $x \equiv K S$.

We shall only be using the logarithmic derivative functions for $l \leq 2-3$ and, for not too small values of $x^{2}$, they may be found by for eward recursion from 


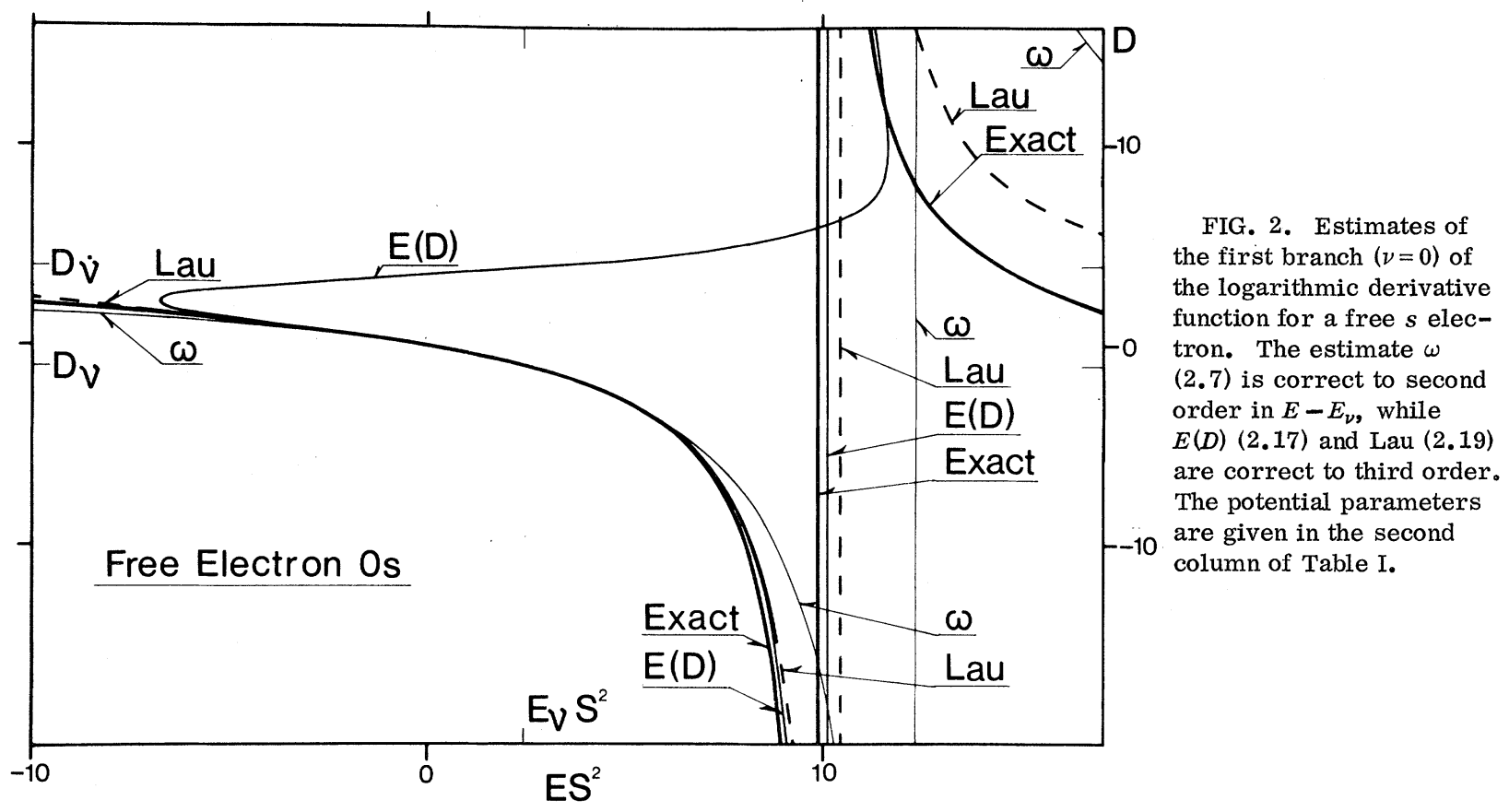

$$
\left[\tilde{D}_{l+1}+(l+1)+1\right]\left(\tilde{D}_{l}-l\right)=-x^{2},
$$

starting with $\tilde{D}_{0}=x \cot x-1$. The parameters $\tilde{\phi}$, $\partial \tilde{\phi} / \partial E$, and $\partial^{2} \tilde{\phi} / \partial E^{2}$ may be obtained by differentiations with respect to energy of the logarithmic derivative function since, from (2.18),

$$
S \phi^{2}(E)=[-\dot{D}(E)]^{-1} \text {. }
$$

Moreover, the radial Schrödinger equation may be written in the form

$$
-S \frac{\partial}{\partial S} D_{l}=\left(D_{l}+l+1\right)\left(D_{l}-l\right)+(E-v) S^{2},
$$

and for free electrons $(E-v) S^{2}=x^{2}$ and

$$
\frac{S \partial}{\partial S}=2 x S^{-2} \frac{\partial}{\partial E} \text {. }
$$

Consequently,

$$
\begin{aligned}
& S \tilde{\phi}^{2}=2 K^{2} /\left[x^{2}+(\tilde{D}+l+1)(\tilde{D}-l)\right], \\
& \frac{\partial \ln \tilde{\phi}}{\partial E}=\frac{3+2 \tilde{D}-S^{3} \tilde{\phi}^{2}}{4 K^{2}}
\end{aligned}
$$

and

$$
-\frac{\partial^{2} \ln \tilde{\phi} / \partial E^{2}}{\partial \ln \tilde{\phi} / \partial E}=\left[2+S^{3} \tilde{\phi}^{2}+\left(S \tilde{\phi} \frac{\partial \tilde{\phi}}{\partial E}\right)^{-1}\right] / 2 K^{2} .
$$

According to (2.30) and (2.32), the free-electron parameters become particularly simple when $D_{\nu l}$ $=l$ or $-l-1$. Hence, $S K_{\nu l}$ is the $\nu$ th zero of the spherical Bessel function $j_{l+1}(x)$ in the former case and the $(\nu+1)$ st zero of $j_{l-1}(x)$ in the latter case, and in both cases $S^{3} \tilde{\phi}_{\nu l}^{2}=2$, provided $K_{\nu l} \neq 0$.
For small values of $x$, the spherical Bessel functions are proportional to $x_{l}$, whereby the logarithmic derivative functions tend towards $l$. For $D_{\nu l}=l$ and $\nu=0$ we may then use (2.30) in reverse to express the coefficients of the Laurent series (2.19) for $\left(\tilde{D}_{l}-l\right)^{-1}$ in terms of the coefficients of the Taylor series for $\tilde{D}_{l+1}+(l+1)+1$. The result is

$$
\begin{aligned}
& S^{3} \tilde{\phi}_{0}^{2}=2 l+3, \\
& -S \tilde{\phi}_{0} \frac{\partial \tilde{\phi}_{0}}{\partial E}=(2 l+5)^{-1},
\end{aligned}
$$

and

$$
S^{-1} \tilde{\phi}_{0}^{2}\left\langle\left(\frac{\partial \tilde{\phi}_{0}}{\partial E}\right)^{2}\right\rangle=\left[(2 l+5)^{2}(2 l+7)\right]^{-1} .
$$

One obvious application of these values is to the computation of $\tilde{D}_{l}\left(K^{2}\right)$ and $S \tilde{\phi}^{2}\left(K^{2}\right)$ for small values of $x^{2}$ where the forward recursion with $(2.30)$ is unstable.

The free-electron potential parameters for $\nu=0$ and $D_{\nu l}=l$ and $-l-1$, respectively, are shown in Table I.

\section{LINEAR-APW METHOD}

We shall now derive a linear reciprocal-space method for solving the band-structure problem. We first consider a periodic muffin-tin potential with one atom per primitive cell, and later mention a simple but approximate way of including non-MT contributions to the potential. (The extension of the formalism to several atoms per cell proceeds exactly as in any other reciprocal-space representation.) Inside the MT spheres the Hamiltonian is 
TABLE I. Potential parameters for free electrons.

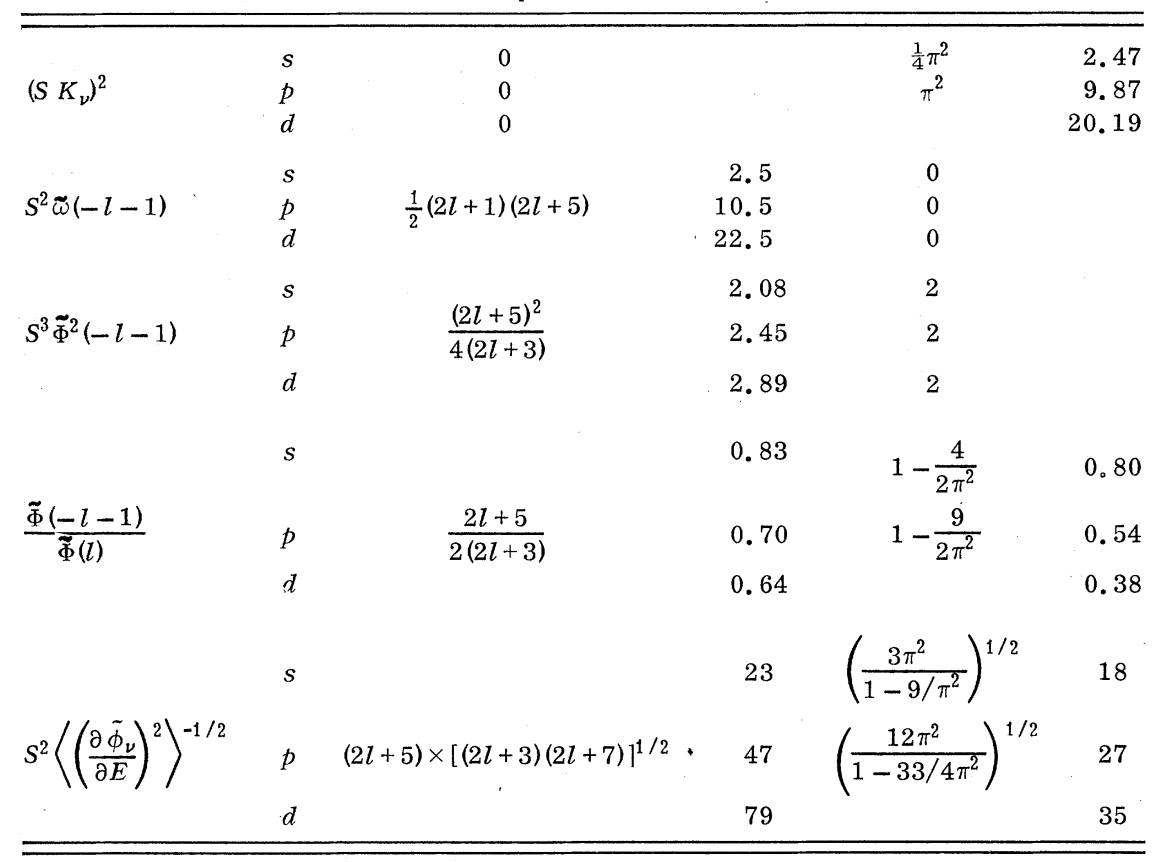

the one considered inSec. $\Pi$, and in the interstitial region the potential assumes the constant value $V_{\mathrm{mtz}}$.

\section{A. LAPW formalism.}

For the solid we choose a set of basis functions, named energy-independent augmented plane waves, and specified by the reciprocal-lattice vectors $\vec{G}$. An energy-independent APW is the plane wave $e^{i(\overrightarrow{\mathrm{k}}+\overrightarrow{\mathrm{G}}) \cdot \overrightarrow{\mathrm{r}}}$ in the interstitial region and, inside any sphere, it is that linear combination of the functions $\Phi_{l m}(D, \overrightarrow{\mathrm{r}})$, defined in (2.2) and (2.3), which matches continuously and differentiably onto the plane wave. In the sphere surrounding the atom at the origin, the energy-independent APW is therefore

$$
\psi_{\overrightarrow{\mathrm{K}}}(\overrightarrow{\mathrm{r}}) \equiv \sum_{l m} 4 \pi Y_{l m}^{*}(\hat{K}) j_{l}(K S) \frac{\Phi_{l m}(\tilde{D}, \overrightarrow{\mathrm{r}})}{\Phi_{l}(\tilde{D})}
$$

where $\overrightarrow{\mathrm{K}}=\overrightarrow{\mathrm{k}}+\overrightarrow{\mathrm{G}}$ is the sum of the Bloch vector and a reciprocal-lattice vector, and $\tilde{D} \equiv \tilde{D}_{l}\left(K^{2}\right)$ is the log- arithmic derivative at the MT sphere for a free electron of angular momentum $l$ and wave number $K$, as defined in (2.29). The definition (3.1) would be that of an ordinary APW if $\Phi\left(\tilde{D}\left(K^{2}\right), r\right)$ were substituted by $\phi(E, r)$. Although the energy-independent APWs are not, individually, solutions at any energy of Schrödinger's equation in the spheres, they must, for the MT potential considered, form a complete set of functions in the neighborhood of the arbitrary energy $E_{\nu}$. In the conventional APW method the variational freedom, arising from the overcompleteness of the plane-wave set in the interstitial region, is used to remove the kink in the individual APW at the sphere. This freedom is used in the linear-APW method to form, inside the sphere and for each value of angular momentum, the linear combination $\Phi(D(E), r)$ corresponding to the proper energy.

In the representation of energy-independent APWs the Hamiltonian and overlap matrices for the MT potential may be obtained from (3.1), (2.4), and (2.6) as

$$
\begin{aligned}
H \underset{\overrightarrow{\mathrm{G}}^{\prime} \overrightarrow{\mathrm{G}}}{\overrightarrow{\mathrm{k}}}-\epsilon O O_{\overrightarrow{\mathrm{G}}^{\prime} \overrightarrow{\mathrm{G}}}^{\overrightarrow{\mathrm{k}}}= & \Omega^{-1}\left\{\psi_{\overrightarrow{\mathrm{K}}^{\prime}}|H-E| \psi_{\overrightarrow{\mathrm{K}}}\right\}=\Omega^{-1}\left\{e^{i \overrightarrow{\mathrm{K}}^{\prime} \cdot \overrightarrow{\mathrm{r}}}\left|-\nabla^{2}+V_{\mathrm{mtz}}-E\right| e^{i \overrightarrow{\mathrm{K}} \cdot \overrightarrow{\mathrm{r}}}\right\} \\
& -\Omega^{-1}\left\langle e^{i \overrightarrow{\mathrm{K}}^{\prime} \cdot \overrightarrow{\mathrm{r}}}\left|-\nabla^{2}+V_{\mathrm{mtz}}-E\right| e^{i \overrightarrow{\mathrm{K}} \cdot \overrightarrow{\mathrm{r}}}\right\rangle+\Omega^{-1}\left\langle\psi_{\overrightarrow{\mathrm{K}}^{\prime}}|H-E| \psi_{\overrightarrow{\mathrm{K}}}\right\rangle \\
= & \left(\delta_{\overrightarrow{\mathrm{G}}^{\prime} \overrightarrow{\mathrm{G}}}-\Omega^{-1}\left\langle e^{i\left(\overrightarrow{\mathrm{G}}-\overrightarrow{\mathrm{G}}^{\prime}\right) \cdot \overrightarrow{\mathrm{r}}}\right\rangle\right)\left(K^{2}+V_{\mathrm{mtz}}-E\right)+\sum_{t} W_{l}\left(\overrightarrow{\mathrm{K}}^{\prime}, \overrightarrow{\mathrm{K}}\right) \Upsilon_{l}\left(K^{\prime}, K, E\right),
\end{aligned}
$$

where $\overrightarrow{\mathrm{K}}^{\prime}=\overrightarrow{\mathrm{k}}+\overrightarrow{\mathrm{G}}^{\prime}$ and $\overrightarrow{\mathrm{K}}=\overrightarrow{\mathrm{k}}+\overrightarrow{\mathrm{G}}$. \langle\rangle denotes an integral in the sphere while \{\} denotes an integral in the cell of volume $\Omega$. Furthermore, 


$$
\begin{aligned}
\Omega^{-1}\left\langle e^{i\left(\overrightarrow{\mathrm{G}}-\overrightarrow{\mathrm{G}}^{\prime}\right) \cdot \overrightarrow{\mathrm{r}}}\right\rangle & =\frac{j_{1}\left(\left|\overrightarrow{\mathrm{G}}-\overrightarrow{\mathrm{G}}^{\prime}\right| S\right)}{\left|\overrightarrow{\mathrm{G}}-\overrightarrow{\mathrm{G}}^{\prime}\right| S} \frac{4 \pi S^{3}}{\Omega} \\
& =\sum_{l} W_{l}\left(\overrightarrow{\mathrm{K}}^{\prime}, \overrightarrow{\mathrm{K}}\right),
\end{aligned}
$$

as obtained from Green's second identity, and the definition

$$
\begin{aligned}
W\left(\overrightarrow{\mathrm{K}}^{\prime}, \overrightarrow{\mathrm{K}}\right) \equiv & (2 l+1) P\left(\hat{K}^{\prime} \cdot \hat{K}\right) j\left(K^{\prime} S\right) j(K S) \\
& \times \frac{\tilde{D}^{\prime}-\tilde{D}}{K^{2}-K^{\prime 2}} \frac{4 \pi S}{\Omega},
\end{aligned}
$$

where we have dropped the subscripts $l$, and where $P$ is the Legendre polynomial. The potential function in (3.2) is

$$
\begin{aligned}
\Upsilon\left(K^{\prime}, K, E\right) & \equiv \frac{K^{2}-K^{\prime 2}}{\tilde{D}^{\prime}-\tilde{D}} \frac{\omega-\epsilon \beta}{S \Phi \Phi^{\prime}} \\
& =\frac{K^{2}-K^{\prime 2}}{\omega-\omega^{\prime}}(\omega-\epsilon \beta),
\end{aligned}
$$

where we have used (2.14) and the notation

$$
\begin{array}{llll}
\epsilon=E-E_{\nu} & \text { and } & & \beta \equiv 1+\left\langle\dot{\phi}_{\nu}^{2}\right\rangle \omega \omega^{\prime}, \\
\omega=\omega_{l}(\tilde{D}) & \text { and } & \omega^{\prime} \equiv \omega_{l}\left(\tilde{D}^{\prime}\right), \\
\Phi \equiv \Phi_{l}(\tilde{D}) & \text { and } & \Phi^{\prime} \equiv \Phi_{l}\left(\tilde{D}^{\prime}\right), \\
\tilde{D} \equiv \tilde{D}_{l}\left(K^{2}\right) & \text { and } & \tilde{D}^{\prime} \equiv \tilde{D}_{l}\left(K^{\prime 2}\right) .
\end{array}
$$

The interpretation of the LAPW matrix (3.2) is straightforward. For a diagonal element, the first term is just $\left(\Omega_{I} / \Omega\right)\left(K^{2}+V_{\mathrm{mtz}}-E\right)$ and, in the second term,

$$
\sum_{l} W(\overrightarrow{\mathrm{K}}, \overrightarrow{\mathrm{K}})=\frac{\Omega_{S}}{\Omega},
$$

where $\Omega_{I}$ and $\Omega_{S}$ are, respectively, the volumes of the interstitial and spherical regions. A diagonal element of the potential function is

$$
\Upsilon(K, K, E)=\tilde{\phi}^{2}\left(K^{2}\right) \frac{1+\left\langle\dot{\phi}_{\nu}^{2}\right\rangle \omega^{2}}{\Phi^{2}\left(\tilde{D}\left(K^{2}\right)\right)}\left[E\left(\tilde{D}\left(K^{2}\right)\right)-E\right],
$$

where we have used (2.31) and (2.17). From the requirement that the diagonal element vanishes, we then realize that, for a single energy-independent APW, the expectation value of the band structure in the extended-zone scheme is the weighted mean of the contribution $K^{2}+V_{\mathrm{mtz}}$ from the interstitial region and the partial energies $E_{l}\left(\tilde{D}_{l}\left(K^{2}\right)\right)$ contributed by the sphere. These partial energies correspond to the boundary conditions at the sphere, defined by the free-space solutions with angular momenta $l$ and the kinetic energy $K^{2}$. The weights are, of course, the respective probabilities for the energyindependent APW state of being in the interstitial region and of being in the sphere with angular momentum $l$. In the approximation that the MT sphere is substituted by the atomic sphere of volume $\Omega$, the weights in the atomic sphere, relative to the free-electron weights $W_{l}$, are approximately the partial band masses

$$
\left.\mu\left(\tilde{D}\left(K^{2}\right)\right) \equiv \frac{d K^{2}}{d \tilde{D}\left(K^{2}\right)} \frac{d D(E)}{d E}\right|_{D(E)=\tilde{D}\left(K^{2}\right)} \approx \frac{\tilde{\phi}^{2}\left(K^{2}\right)}{\phi^{2}(E(\tilde{D}))}
$$

relative to the mass of a free electron. For the transition metal Pd, we demonstrate in Fig. 3 how its $4 d$ mass, which is eight times that of free electrons, causes the single LAPW band to follow the $4 d$ energy in the region of $K S$ values where $W_{d}$ dominates.

We may rearrange the terms of (3.2) in such a way that each term becomes Hermitian, the $l$ convergence is improved, and the LAPW matrix takes the form

$$
\begin{aligned}
H \overrightarrow{\mathrm{G}}_{\overrightarrow{\mathrm{G}}^{\prime}}^{\overrightarrow{\mathrm{G}}}-\epsilon O_{\overrightarrow{\mathrm{G}}}^{\overrightarrow{\mathrm{k}}} \cdot \overrightarrow{\mathrm{G}}= & \left(K^{2}-E\right) \delta_{\overrightarrow{\mathrm{G}}^{\prime} \overrightarrow{\mathrm{G}}^{\prime}}+V\left(\overrightarrow{\mathrm{G}}^{\prime}-\overrightarrow{\mathrm{G}}\right) \\
& +V_{S}\left(\overrightarrow{\mathrm{K}}^{\prime}, \overrightarrow{\mathrm{K}}, E\right)
\end{aligned}
$$

familiar from OPW and pseudopotential theory. The contribution to the pseudopotential from the interstitial region is

$$
V\left(\overrightarrow{\mathrm{G}}^{\prime}-\overrightarrow{\mathrm{G}}\right)=V_{\mathrm{mtz}}\left(\delta_{\overrightarrow{\mathrm{G}}^{\prime} \overrightarrow{\mathrm{G}}}-\Omega^{-1}\left\langle e^{i\left(\overrightarrow{\mathrm{G}}-\overrightarrow{\mathrm{G}}^{\prime}\right) \cdot \overrightarrow{\mathrm{r}}}\right\rangle\right),
$$

while the contribution from the sphere is

$$
\begin{aligned}
V_{S}\left(\overrightarrow{\mathrm{K}}^{\prime}, \overrightarrow{\mathrm{K}}, E\right)= & \Omega^{-1}\left\langle\psi_{\overrightarrow{\mathrm{K}}^{\prime}}|H-E| \psi_{\overrightarrow{\mathrm{K}}}\right\rangle \\
& -\Omega^{-1}\left\langle e^{i \overrightarrow{\mathrm{K}}^{\prime} \cdot \overrightarrow{\mathrm{r}}}\left|-\nabla^{2}-E\right| e^{i \overrightarrow{\mathrm{K}} \cdot \overrightarrow{\mathrm{r}}}\right\rangle \\
= & \sum_{l} W\left(\overrightarrow{\mathrm{K}}^{\prime}, \overrightarrow{\mathrm{K}}\right)\left[\Gamma\left(K^{\prime}, K\right)-\epsilon \Delta\left(K^{\prime}, K\right)\right],
\end{aligned}
$$

where we have used (3.3). Moreover,

$$
\begin{aligned}
& \Gamma\left(K^{\prime}, K\right)=\left(\omega^{\prime} \lambda-\omega \lambda^{\prime}\right) /\left(\omega-\omega^{\prime}\right), \\
& \Gamma(K, K)=\omega \tilde{\phi}^{2}\left(K^{2}\right) / \Phi^{2}-\lambda, \\
& \Delta\left(K^{\prime}, K\right)=\left[\left(\omega^{\prime}-\beta \lambda^{\prime}\right)-(\omega-\beta \lambda)\right] /\left(\omega-\omega^{\prime}\right), \\
& \Delta(K, K)=\tilde{\phi}^{2}\left(K^{2}\right)\left(1+\left\langle\dot{\phi}_{\nu}^{2}\right\rangle \omega^{2}\right) / \Phi^{2}-1,
\end{aligned}
$$

with the notation

$$
\lambda \equiv K^{2}-E_{\nu} \quad \text { and } \quad \lambda^{\prime} \equiv K^{\prime 2}-E_{\nu} .
$$

For the low values of $l$ needed in these expressions, the free-electron parameters $\tilde{D}_{l}\left(K^{2}\right)$ and $S \tilde{\phi}_{l}^{2}\left(K^{2}\right)$ may be obtained from (2.30) and (2.32) or, for small values of $K^{2}$, from the Laurent series around $K=0$, as is described in Sec. II D. The potential functions $\omega_{l}(\tilde{D})$ and $\Phi_{l}(\tilde{D})$ may then be obtained from (2.12) and (2.13).

\section{B. Accuracy and convergence}

We expect the LAPW method to yield energy bands correct to order $\epsilon^{3}$, as is the case for a single sphere. This will now be proved for a constant po- 
tential and, since this is no simpler than any other MT potential when treated with a linear method, we believe the result to be true in general.

We take the constant potential as the zero of energy so that $V\left(\vec{G}^{\prime}-\vec{G}\right)$ vanishes. The potential parameters are now the free-electron parameters described in Sec. II D and, from the relation (2.24) between $\tilde{\omega}\left(\tilde{D}\left(K^{2}\right)\right)$ and $\lambda$, we realize that the leading term of the pseudopotential component $\Gamma-\epsilon \Delta$ becomes

$$
-\left[\left(\lambda+\lambda^{\prime}\right) \lambda \lambda^{\prime}-\epsilon\left(\lambda^{2}+\lambda^{\prime 2}\right)\right]\left\langle\left(\frac{\partial \tilde{\phi}_{\nu l}}{\partial E}\right)^{2}\right\rangle .
$$

This is of higher order than the exact term $(\lambda-\epsilon) \delta_{\vec{G}} \vec{G}$ in (3.8). We may then use perturbation theory and put $\epsilon=\lambda$ in (3.15). The diagonal element of the pseudopotential is now of order $\lambda^{4}$, while the off-diagonal elements are of order $\lambda^{2}$ and, consequently, the LAPW energies are correct to third order while the eigenvectors are correct to first order.

The convergence of the sum on angular momentum in (3.10) and the strength of the pseudopotential depend on the choice of energy zero in the first, free-electron term of (3.8). With increasing angular momenta, the potential parameters approach those of that constant potential which coincides with the true potential at the sphere and, if the zero of energy is taken at this value, the pseudopotential components of high angular momenta will vanish as (3.15). This happens in practice when $l \geq 2-3$.

Among those reciprocal-space methods that can handle transition-metal $d$ bands, the APW method seems to have the best $\vec{G}$ convergence, and in that case the trial function must include all plane waves with a significant $d$ contribution $W_{d}$ in order to reproduce the proper angular behavior in the interstitial region. The corresponding wave numbers satisfy $K S \leq 6-7$, when $S$ is the atomic-sphere radius. From the discussion of (3.6) and (3.7), this criterion also applies to the LAPW method (although the eigenvectors of the two methods are not necessarily identical to order $\epsilon$, because a plane-wave expansion in the interstitial region is not unique). From Fig. 3, it appears that the 25-50 energy-independent APW's, which for a given Bloch vector have $K S \lesssim 6-7$, are nearly degenerate and they must therefore be simultaneously included in the basis set. The high values attained by the energies beyond $K S=7$ indicate that at this point the LAPW matrix is converged in the low-energy region, which includes the $d$ band. A complete application of the LAPW method to the band structure of $\mathrm{Cu}$ is presented by Koelling and Arbman in Ref. 18.

\section{Non-MT contributions}

Non-MT contributions to the potential are only important in the outer region of the MT sphere and in the interstitial region, and, since the energy-in-

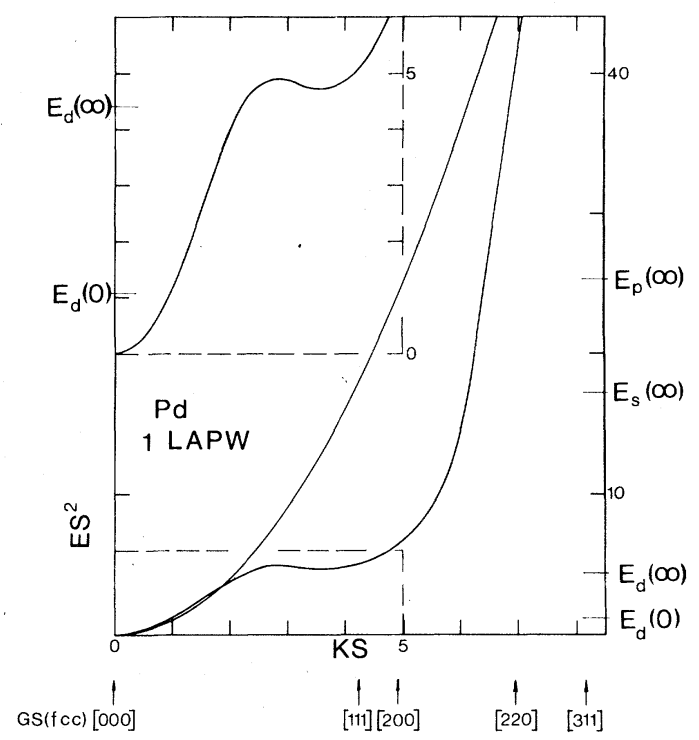

FIG. 3. Expectation value of the band structure of palladium (Ref。26) using a single energy-independent APW. Atomic sphere radius is $2.873 \mathrm{Bohr}$ radii. Distances to the nearest reciprocal-lattice points are indicated at the bottom. Extension of $d$ band is from $E_{d}(0)$ to $E_{d}(\infty)$.

dependent APW is continuous and differentiable at the sphere, these non-MT contributions can usually be incorporated simply by adding their Fourier components $\Delta V\left(\overrightarrow{\mathrm{G}}^{\prime}-\overrightarrow{\mathrm{G}}\right)$ to (3.9). If, however, the $l^{0}$ th partial band mass (3.7) differs substantially from unity, we must, in addition to $\Delta V\left(\overrightarrow{\mathrm{G}}^{\prime}-\overrightarrow{\mathrm{G}}\right)$, and together with $\sum_{\imath} W \Gamma$ in (3.10), include the contributions involving $\left\langle\Phi_{l^{\prime} m^{\prime}}^{\prime}|\Delta V| \Phi_{l} 0_{m}\right\rangle$ and $\left\langle j_{l}{ }^{\prime} \cdot|\Delta v| j_{l} 0_{m}\right\rangle$. Owing to the fast convergence of (3.10), these matrices are diagonal in $l$ for cubic transition metals.

\section{Comparison with APW formalism}

It was previously mentioned that an ordinary APW is given by $(3.1)$ if $\Phi\left(\tilde{D}\left(K^{2}\right), r\right)$ is substituted by $\phi(E, r)$. Moreover, the APW matrix may be expressed by (3.2) if, in (3.5), we perform the substitution

$$
[\omega(\tilde{D})-\epsilon \beta] / S \Phi(\tilde{D}) \Phi\left(\tilde{D}^{\prime}\right) \rightarrow D(E)-\tilde{D}\left(K^{2}\right),
$$

whereby the reverse transition, from the APW to the LAPW matrix, is formally a substitution of $D(E)$ by its first-order expansion about $\tilde{D}\left(K^{2}\right)$. Hence, in the LAPW method, the rapidly varying logarithmic derivative functions are substituted by linear functions of energy, and there are no difficulties near the asymptotes $E_{n l}(\infty)$ of the logarithmic derivative functions as in the ordinary APW method. For comparison with the single-LAPW result of Fig. 3, we show in Fig. 4 the expectation value of the Pd band structure using a single APW. Such single-APW bands were used in the 1953 ver- 


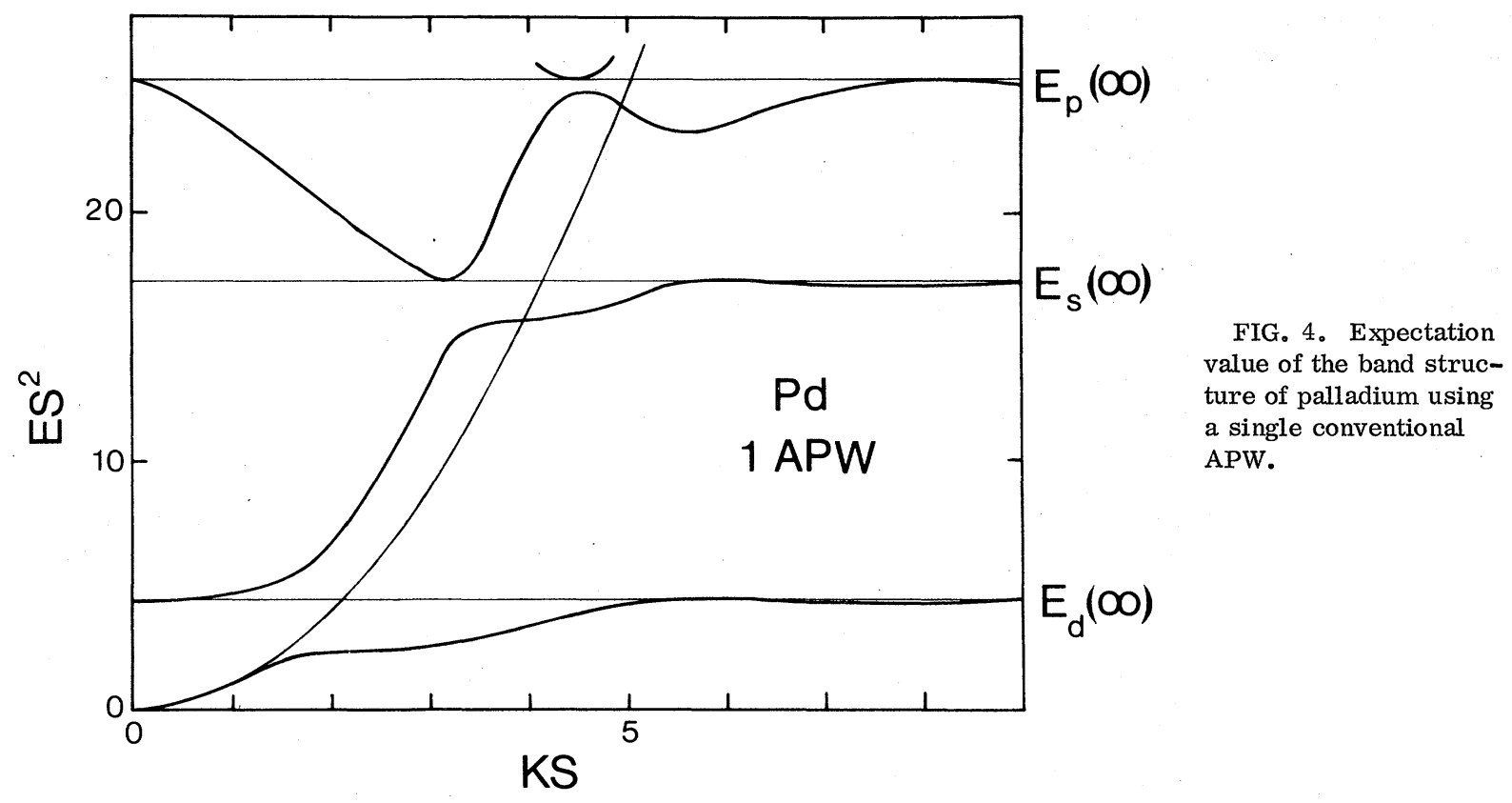

sion of the APW method, as we shall discuss below. This band does not have the simple appearance of the LAPW band; it has a branch for each value of $n l$, and the branches are separated by the asymptotes $E_{n l}(\infty)$. The band touches the $n l$ asymptotes at the free-electron asymptotes $K^{2}=\tilde{E}_{n^{\prime} l}(\infty)$, and, even for free electrons, the single-APW band will consist of individual branches sticking together at the free-electron parabola.

The APW pseudopotential appears from (3.8) and (3.10) when the linear function $\Gamma-\epsilon \Delta$ is substituted by

$$
\frac{\left(D-\tilde{D}^{\prime}\right)\left(K^{2}-E\right)-(D-\tilde{D})\left(K^{\prime 2}-E\right)}{\tilde{D}^{\prime}-\tilde{D}},
$$

with $D \equiv D(E)$. This expression is a rapidly varying function of energy, diverging at $E_{n l}(\infty)$, and this is true even for free electrons where the APW pseudopotential vanishes along $K^{\prime 2}=E$ and $K^{2}=E$ only, rather than in a region of $\left(K^{\prime 2}, K^{2}, E\right)$ space. At a particular energy, e.g., the Fermi energy $E_{F}$, not coinciding with any of the asymptotes, the APW pseudopotential is specified by the parameters $D_{l}\left(E_{F}\right)$ and $V_{\mathrm{mtz}},{ }^{9}$ while the LAPW pseudopotential, with $E_{\nu}=E_{F}$, depends on the additional parameters $\dot{\phi}_{l}\left(E_{F}\right)$. These parameters only affect the bands away from the Fermi energy and, if they are set equal to zero, the LAPW pseudopotential equals to the APW pseudopotential. This may be seen from (3.11), (2.7), and (2.8).

In the 1953 version of the APW method, ${ }^{23}$ the basis functions are in fact energy-independent APWs but of a more complicated nature than the ones considered above. They are APWs of wave vector $\overrightarrow{\mathrm{K}}=\overrightarrow{\mathrm{k}}+\overrightarrow{\mathrm{G}}$, and with $\Phi_{l}(r)$ in (3.1) substituted by $\phi_{l}\left(E_{n}^{K}, r\right)$. The $E_{n}^{K}$ are the single-APW energies illustrated in Fig. 4, and they are not the partial energies $E_{n l}\left(\tilde{D}_{l}\left(K^{2}\right)\right)$ that make the basis functions differentiable. The basis set carries the double index $n \overrightarrow{\mathrm{G}}$ and, in practice, those functions corresponding to the first few branches of $E_{n}^{K}$ together with all the reciprocal-lattice points used in the APW and LAPW methods must be included. Hence, the dimension of the eigenvalue problem is two or three times larger than that of the LAPW method. Moreover, the single-APW eigenvalues $E_{n}^{K}$ cannot be given explicitly and their evaluation is difficult.

\section{LINEAR-MTO METHOD}

In this section we present a linear band-structure method in the angular momentum representation which is particularly suited for closely packed structures. For the angular functions we often use the general notation

$$
Y_{L}(\hat{r}) \equiv \sum_{m=-l}^{l} Y_{l m}(\hat{r}) U_{l m ; L}^{*},
$$

where $L$ is short for $l u$ and $U_{m ; u}$ is an arbitrary unitary matrix of dimension $(2 l+1)$. We again consider the periodic MT potential with one atom per primitive cell and, for convenience, we choose the atomic positions to coincide with the lattice translations $\vec{R}$, although in Sec. IV F we shall extend the formalism to more than one atom per cell.

\section{A. Energy-independent MTO}

The energy-independent MTO $\chi_{L}(\overrightarrow{\mathrm{r}})$ has a tail proportional to $r^{-l-1} i^{l} Y_{L}(\hat{r})$ in the interstitial region. 
This part of the tail is a solution of the Laplace equation, $\nabla^{2} \chi=0$, rather than a solution of the Helm-: holtz wave equation, $\left(\nabla^{2}+E-V_{\mathrm{mtz}}\right) \chi=0$, used for the exact MTO, ${ }^{12}$ and the present choice leads to important simplifications of the KKR structure constants but also to small errors, of order $\left(E-V_{\mathrm{mtz}}\right)^{2}$, in the computed energy bands The energy-independent MTO is everywhere continuous and differentiable. Inside its own sphere it equals $\Phi_{L}(-l-1$, $\vec{r})$, as defined by (2.3) and (4.1), and inside any other sphere, say the one displaced by $\vec{R}$, it is the linear combination of the $\Phi_{L},\left(l^{\prime}, \overrightarrow{\mathrm{r}}-\overrightarrow{\mathrm{R}}\right)$ 's that matches on to $\chi_{L}(\vec{r})$. Apart from the normalization, the coefficients of this linear combination are then the coefficients in the expansion about $\vec{R}$ of the $2^{l}$-pole field $r^{-t-1} i^{\imath} Y_{L}(\hat{r})$ in the regular solutions $|\overrightarrow{\mathrm{r}}-\overrightarrow{\mathrm{R}}|^{l^{\prime}} i^{l^{\prime}} Y_{L} \cdot(\overrightarrow{\mathrm{r}}=\overrightarrow{\mathrm{R}})$ of the Laplace equation.

The Bloch sum of orbitals

$$
\chi_{L}^{\overrightarrow{\mathrm{k}}}(\overrightarrow{\mathrm{r}}) \equiv \sum_{\overrightarrow{\mathrm{R}}} e^{i \overrightarrow{\mathrm{k}} \cdot \overrightarrow{\mathrm{R}}} \chi_{L}(\overrightarrow{\mathrm{r}}-\overrightarrow{\mathrm{R}})
$$

has an expansion about the origin that may be written as

$$
\begin{aligned}
\chi_{L}^{\overrightarrow{\mathbf{k}}}(\overrightarrow{\mathbf{r}})= & \Phi_{L}(-l-1, \overrightarrow{\mathbf{r}}) \\
& -\sum_{L^{\prime}} \frac{\Phi_{L^{\prime}}\left(l^{\prime}, \overrightarrow{\mathbf{r}}\right)}{2\left(2 l^{\prime}+1\right)} \frac{\Phi_{l}(-l-1)}{\Phi_{l^{\prime}}\left(l^{\prime}\right)} \mathrm{s}_{L^{\prime}}^{\overrightarrow{\mathbf{k}}},
\end{aligned}
$$

and which is valid in the sphere at the origin. The first term is the contribution from $\vec{R}=\overrightarrow{0}$ in (4.2) and the second term is the sum of the tails coming from all other sites in the crystal. The structure constants $S_{L}^{\overrightarrow{\mathrm{k}}}{ }_{L}$ in (4.3) form a Hermitian matrix, they may be defined by the one-center expansion

$$
\begin{aligned}
& \sum_{\overrightarrow{\mathrm{R}} \neq 0} e^{i \overrightarrow{\mathrm{k}} \cdot \overrightarrow{\mathrm{R}}} \frac{S^{l+1}}{|\overrightarrow{\mathrm{r}}-\overrightarrow{\mathrm{R}}|^{l+1}} i^{l} Y_{l}(\overrightarrow{\mathrm{r}}-\overrightarrow{\mathrm{R}}) \\
& \quad=-\sum_{L^{\prime}} \frac{1}{2\left(2^{\prime}+1\right)} \frac{r^{l^{\prime}}}{S^{l^{\prime}}} i^{l^{\prime}} Y_{L} \cdot(\hat{r}) S_{L^{\prime}{ }^{\overrightarrow{\mathrm{k}}},},
\end{aligned}
$$

and we shall return to them in Sec. IVD.

In the basis of the Bloch sums (4.2), the contribution to the Hamiltonian and overlap matrices from all the spheres are just the integrals in the sphere at the origin, as given by (2.4) and (2.6) in connection with the one-center expansion (4.3). Hence, when we neglect the contributions from the interstitial region, the one-center energies, stemming from the first term in (4.3), will be $(2 l+1)$ degenerate and equal to $E_{l}(-l-1)$, as given by (2.17). It will prove convenient, however, to write the onecenter expansion in a more general form by adding in (4. 3) some constant times $\Phi_{L}(l, \vec{r})$ to the first term and subtracting it in the tail sum. If, at the same time, we renormalize the orbital, the resulting first term may, according to (2.11), be written as $\Phi_{L}\left(D_{L}, \overrightarrow{\mathrm{r}}\right)$, where $D_{L}$ is an arbitrary logarithmic derivative, but different from $l$, and the formal onecenter energies become $E_{l}\left(D_{L}\right)$. Hence, with the normalization

$$
\chi_{L}\left(D_{L}, \overrightarrow{\mathrm{r}}\right) \equiv \frac{\omega_{l}\left(D_{L}\right)-\omega_{l}(l)}{\omega_{l}(-l-1)-\omega_{l}(l)} \chi_{L}(\overrightarrow{\mathrm{r}})
$$

or equivalently

$$
(2 l+1) \chi_{l}\left(D_{L}\right)=\left(l-D_{L}\right) \Phi_{l}\left(D_{L}\right),
$$

the general form of the one-center expansion is

$$
\chi_{L}^{\overrightarrow{\mathrm{k}}}\left(D_{L}, \overrightarrow{\mathbf{r}}\right)=\Phi_{L}\left(D_{L}, \overrightarrow{\mathbf{r}}\right)-\sum_{L} \frac{\Phi_{L} \cdot\left(l^{\prime}, \overrightarrow{\mathbf{r}}\right)}{\left.\omega_{l^{\prime}}\left(D_{L}\right)^{\prime}\right)-\omega_{l^{\prime}}\left(l^{\prime}\right)} T_{L}^{\overrightarrow{\mathrm{k}}}{ }_{L} .
$$

We have used (2.12)-(2.15) in (4.6) and we have defined a Hermitian matrix which may be written as

$$
\begin{gathered}
T_{L}^{\overrightarrow{\mathrm{k}}{ }_{L}}\left(D_{L^{\prime}}, D_{L}\right) \equiv\left(\sqrt{\frac{1}{2}} S\right) \chi_{\chi_{l}},\left(D_{L}{ }^{\prime}\right)\left\{-2(2 l+1)\left[\left(D_{L}\right.\right.\right. \\
\left.\left.+l+1) /\left(D_{L}-l\right)\right] \delta_{L^{\prime}{ }_{L}}+\delta_{{ }^{\prime}{ }^{\prime}{ }_{L}}^{\overrightarrow{\mathrm{k}}}\right\}\left(\sqrt{\frac{1}{2}} S\right) \chi_{\chi_{l}}\left(D_{L}\right) . \\
\text { B. Hamiltonian and overlap matrices }
\end{gathered}
$$

\section{B. Hamiltonian and overlap matrices}

The spherical contributions to the Hamiltonian and overlap matrices in the basis of $\chi_{L}^{\overrightarrow{\mathbf{k}}}\left(D_{L}, \overrightarrow{\mathbf{r}}\right)$ are now

$$
\begin{aligned}
& \left\langle\chi_{L}^{\overrightarrow{\mathbf{k}}} \cdot\left|H-E_{\nu}\right| \chi_{L}^{\overrightarrow{\mathbf{k}}}\right\rangle=\omega_{l}\left(D_{L}\right) \delta_{L}^{\prime} L-\left(1+\frac{\omega_{l} \cdot\left(l^{\prime}\right)}{\omega_{l} \cdot\left(D_{L^{\prime}}\right)-\omega_{l} \cdot\left(l^{\prime}\right)}+\frac{\omega_{l}(l)}{\omega_{l}\left(D_{L}\right)-\omega_{l}(l)}\right) T_{L}^{\overrightarrow{\mathbf{k}}} \\
& +\sum_{L^{\prime \prime}} T_{L}^{\overrightarrow{\mathbf{k}}{ }^{\prime}}{ }^{\prime \prime} \frac{\omega_{l^{\prime \prime}}\left(l^{\prime \prime}\right)}{\left[\omega_{l}{ }^{\prime \prime}\left(D_{L}{ }^{\prime \prime}\right)-\omega_{l}{ }^{\prime \prime}\left(l^{\prime \prime}\right)\right]^{2}} T_{L}^{\overrightarrow{\mathrm{k}}}{ }^{\prime \prime L}
\end{aligned}
$$

and

$$
\begin{aligned}
& \left\langle\chi_{L}^{\overrightarrow{\mathrm{k}}} \cdot \mid \chi_{L}^{\overrightarrow{\mathrm{k}}}\right\rangle=\left[1+\left\langle\dot{\phi}_{\nu l}^{2}\right\rangle \omega_{l}^{2}\left(D_{L}\right)\right] \delta_{L^{\prime} L}-\left(\frac{1+\left\langle\dot{\phi}_{\nu l}^{2}{ }^{\prime}\right\rangle \omega_{l^{\prime}}\left(D_{L}^{\prime}\right) \omega_{l}\left(l^{\prime}\right)}{\omega_{l^{\prime}}\left(D_{L^{\prime}}\right)-\omega_{l^{\prime}}\left(l^{\prime}\right)}+\frac{1+\left\langle\dot{\phi}_{\nu l}^{2}\right\rangle \omega_{l}\left(D_{L}\right) \omega_{l}(l)}{\omega_{l}\left(D_{L}\right)-\omega_{l}(l)}\right) T_{L}^{\overrightarrow{\mathrm{k}}}{ }_{L} \\
& +\sum_{L} T_{L}^{\overrightarrow{\mathrm{k}} L^{\prime}} \frac{1+\left\langle\dot{\phi}_{\nu l^{\prime \prime}}^{2}\right\rangle \omega_{l}^{2} \cdot\left(l^{\prime \prime}\right)}{\left[\omega_{t^{\prime \prime}}\left(D_{L}^{\prime \prime}\right)-\omega_{l}{ }^{\prime \prime}\left(l^{\prime \prime}\right)\right]^{2}} T_{L}^{\overrightarrow{\mathrm{k}} \prime^{\prime} L}
\end{aligned}
$$


where the choice of $D_{L}$ only affects the normalization and the arrangement of the various terms. If we use $D_{L}=-l-1$, as in (4.3),

$$
T_{L}^{\overrightarrow{\mathrm{k}}{ }^{\prime} L}=\left(\sqrt{\frac{1}{2}} S\right) \Phi_{l} \cdot\left(-l^{\prime}-1\right) S_{L}^{\overrightarrow{\mathrm{k}}}{ }_{L}\left(\sqrt{\frac{1}{2}} S\right) \Phi_{l}(-l-1),
$$

and in this case the terms of order $T^{0}, T^{1}$, and $T^{2}$ in (4.9) and (4.10) may be identified as, respectively, one-, two-, and three-center integrals. For other choices of $D_{L}$ this identification is purely formal.

Before properly computing the integrals in the region between the MT sphere and the atomic polyhedron, we mention that the simplest approximate way of including this interstitial region is to substitute the atomic Wigner-Seitz sphere for the MT sphere in (4.9) and (4.10), that is, both in the structure constants (4.4) and in the potential parameters. We thus emphasize that (4.9) and (4.10) are the important matrices of the LMTO formalism and what we describe in the remainder of this section can merely be regarded as a correction to the atomic-sphere approximation (ASA).

The expansion (4.4) converges inside the sphere passing through the atomic positions nearest to the origin. The atomic polyhedron of any closely packec structure is included in this sphere and an integral in the interstitial region may therefore be computed entirely in the angular momentum representation from this expansion and the expansion in spherical harmonics of the interstitial step function, ${ }^{12}$ which is unity in the interstitial region and zero elsewhere This requires a double sum on $L^{\prime \prime}$ and $L^{\prime \prime \prime}$ and, in order that the accuracy be improved beyond that of the ASA, the sum must include $\left(l^{\prime \prime}, l^{\prime \prime \prime}\right) \lesssim 4$; hence it becomes quite tedious. An integral in the interstitial region could also be computed entirely in the reciprocal-lattice representation by using the second factor of (3.9) for the step function together with a Fourier transform of the MTO tail, but this again requires a double summation. The most convenient way of handling the integral is, however, to evaluate the integral in the cell by a single reciprocal-lattice sum and then to subtract the integral in the sphere by subtractions in (4.9) and (4.10). In this way, the LMTO Hamiltonian and overlap matrices become

$$
\begin{aligned}
H_{L}^{\overrightarrow{\mathrm{k}}{ }_{L}}= & \left\langle\chi_{L}^{\overrightarrow{\mathrm{k}}} \cdot\left|H-E_{\nu}\right| \chi_{L}^{\overrightarrow{\mathrm{k}}}\right\rangle \\
& -\left(V_{\mathrm{mtz}}-E_{\nu}\right)\left\langle\tilde{\chi}_{L}^{\overrightarrow{\mathrm{k}}} \cdot \mid \tilde{\chi}_{L}^{\overrightarrow{\mathrm{k}}}\right\rangle+\left(V_{\mathrm{mtz}}-E_{\nu}\right)\left\{\tilde{\chi}_{L}^{\overrightarrow{\mathrm{k}}} \cdot \mid \tilde{\chi}_{L}^{\overrightarrow{\mathrm{k}}}\right\}
\end{aligned}
$$

and

$$
O_{L}^{\overrightarrow{\mathbf{k}}}{ }_{L}=\left\langle\chi_{L}^{\overrightarrow{\mathbf{k}}} \cdot \mid \chi_{L}^{\overrightarrow{\mathbf{k}}}\right\rangle-\left\langle\tilde{\chi}_{L}^{\overrightarrow{\mathbf{k}}} \cdot \mid \tilde{\chi}_{L}^{\overrightarrow{\mathbf{k}}}\right\rangle+\left\{\tilde{\chi}_{L}^{\overrightarrow{\mathbf{k}}} \cdot \mid \tilde{\chi}_{L}^{\overrightarrow{\mathbf{k}}}\right\},
$$

where \{\} denotes an integral in the cell of volume $\Omega$.

We want the reciprocal-lattice sum to be rapidly convergent and to be given by a convenient analytical expression. Moreover, $\left\langle\tilde{\chi}_{L}^{\overrightarrow{\mathbf{k}}}, \mid \tilde{\chi}_{L}^{\overrightarrow{\mathbf{k}}}\right\rangle$ should have the form of (4.10). The orbital $\tilde{\chi}_{L}(\overrightarrow{\mathrm{r}})$ therefore coincides with the proper orbital in the interstitial region only while, inside the spheres, it is a smooth function derived from a pseudopotential. If we let this potential be constant and equal to $E_{\nu}$, the corresponding potential parameters are the free-electron parameters with $K_{\nu}=0$, derived in Sec. II D and given in the first column of Table $I$. Consequently,

$$
\tilde{\Phi}(l, r)=\tilde{\phi}_{0}(r)=\left[(2 l+3) S^{-3}\right]^{1 / 2}(r / S)^{2},
$$

so that the tail of the free-electron orbital is proportional to $r^{-l-1} i^{l} Y_{L}(\hat{r})$, not only in the interstitial region, but also inside all other spheres, that is,

$$
\tilde{\chi}_{l}(r) \equiv \chi_{l}\left(D_{L}\right) \begin{cases}\tilde{\Phi}_{l}(-l-1, r) / \tilde{\Phi}_{l}(-l-1), \\ (r / S)^{-l-1}, & r \geq S\end{cases}
$$

where the normalization constant depends on the proper orbital and is given by (4.6). The reciprocal-lattice representation of the Bloch sum is

$$
\tilde{\chi}_{L}^{\overrightarrow{\mathrm{k}}}(\overrightarrow{\mathrm{r}})=\chi_{I}\left(D_{L}\right) \sum_{\overrightarrow{\mathrm{G}}} e^{i \overrightarrow{\mathrm{K}} \cdot \overrightarrow{\mathrm{r}}} F_{L}(\overrightarrow{\mathrm{K}})
$$

where $\overrightarrow{\mathrm{K}} \equiv \overrightarrow{\mathrm{k}}+\overrightarrow{\mathrm{G}}$ and

$$
F_{L}(\overrightarrow{\mathrm{K}}) \equiv(2 l+1)(2 l+3) \frac{4 \pi S^{3}}{\Omega} \frac{j_{l+1}(K S)}{(K S)^{3}} Y_{L}(\hat{K}),
$$

as obtained from Green's second identity. The integral in the cell is therefore

$$
\left\{\tilde{\chi}_{L}^{\overrightarrow{\mathrm{k}}}, \mid \tilde{\chi}_{L}^{\overrightarrow{\mathrm{k}}}\right\}=\chi_{l^{\prime}}\left(D_{L^{\prime}}\right) \Omega \mathcal{F}_{L}^{\overrightarrow{\mathrm{k}}{ }_{L}} \chi_{I}\left(D_{L}\right),
$$

with

$$
\Im_{L^{\prime} L}^{\overrightarrow{\mathbf{k}}} \equiv \sum_{\overrightarrow{\mathrm{G}}} F_{L}^{*},(\overrightarrow{\mathrm{K}}) F_{L}(\overrightarrow{\mathrm{K}}) .
$$

In (4.13), the difference between the two integrals in the sphere $\left\langle\chi_{L}^{\overrightarrow{\mathbf{k}}}, \mid \chi_{L}^{\overrightarrow{\mathbf{k}}}\right\rangle-\left\langle\tilde{\chi}_{L}^{\overrightarrow{\mathbf{k}}}, \mid \tilde{\chi}_{L}^{\overrightarrow{\mathbf{k}}}\right\rangle$ is given by (4.10) if, from $1+\left\langle\dot{\phi}_{\nu}^{2}\right\rangle \omega^{2}(D)$ in the formal one-center integral, we subtract

$$
\left[1+\left\langle\left(\frac{\partial \tilde{\phi}_{0}}{\partial E}\right)^{2}\right\rangle \tilde{\omega}^{2}(D)\right]\left(\frac{\omega(D)-\omega(l)}{\tilde{\omega}(D)-\tilde{\omega}(l)}\right)^{2} \frac{\tilde{\Phi}^{2}(l)}{\Phi^{2}(l)}
$$

and, from $1+\left\langle\dot{\phi}_{\nu}^{2}\right\rangle \omega(D) \omega(l)$ in the formal two-center integrals, we subtract

$$
\frac{\omega(D)-\omega(l)}{\tilde{\omega}(D)-\tilde{\omega}(l)}
$$

and, finally, from $1+\left\langle\dot{\phi}_{\nu}^{2}\right\rangle \omega^{2}(l)$ in the formal threecenter integrals, we subtract

$$
\Phi^{2}(l) / \tilde{\Phi}^{2}(l) \text {. }
$$

Similarly in (4.12), the subtraction of $\left(V_{\mathrm{mtz}}-E_{\nu}\right)$ $\times\left\langle\tilde{\chi}_{L}^{\overrightarrow{\mathrm{k}}} \cdot \mid \tilde{\chi}_{L}^{\overrightarrow{\mathrm{k}}}\right\rangle$ from (4.9) may be accomplished by sub- 
tracting $\left(V_{\mathrm{mtz}}-E_{\nu}\right)$ times (4.18) from $\omega(D)$ in the formal one-center integral of (4.9), by subtracting $\left(V_{\mathrm{mtz}}-E_{\nu}\right)$ times (4.19) from $\omega(l)$ in the formal two-center integrals, and by subtracting $\left(V_{\mathrm{mtz}}-E_{\nu}\right)$ times (4.20) from $\omega(l)$ in the formal three-center integrals. When doing so, we have used the same $D_{L}$ in $\chi_{L}\left(D_{L}, \overrightarrow{\mathbf{r}}\right)$ and $\tilde{\chi}_{L}\left(D_{L}, \overrightarrow{\mathbf{r}}\right)$. In (4.18)-(4.20), we have used the subscript $\nu=0$ for the freeelectron parameters and we recall that $\tilde{\omega}(l)=0$ and $S^{3} \tilde{\Phi}^{2}(l)=2 l+3$.

The above-mentioned procedure is analogous to the one followed when writing the LAPW matrix in the form (3.8) and, besides its immediate purpose of including the interstitial region, it has improved the convergence of the sums on $L^{\prime \prime}$ in (4.9) and (4.10) because the components of high angular momenta are free-electron-like and hence included in the Fourier sum (4.17). In practice, the $L^{\prime \prime}$ summations are converged when $l^{\prime \prime} \leq 2-3$. In (4.12) and (4.13), the two last terms, involving $\tilde{\chi}$, will therefore be referred to as the combined correction term.

The orthonormalized wave functions are most conveniently given by one-center expansions, e.g.,

$$
\Psi_{j}^{\overrightarrow{\mathrm{k}}}(\overrightarrow{\mathrm{r}})=\sum_{L} \sum_{L^{\prime}}\left[\phi_{\nu L}(\overrightarrow{\mathrm{r}}) \pi_{L L^{\prime}}^{\overrightarrow{\mathrm{k}}}+\dot{\phi}_{\nu L}(\overrightarrow{\mathrm{r}}) \Omega_{L L^{\prime}}^{\overrightarrow{\mathrm{k}}}\right] A_{L^{\prime}{ }_{j}}^{\overrightarrow{\mathrm{k}}},
$$

where, according to (4.7) and (2.2),

$$
\pi_{L L^{\prime}}^{\overrightarrow{\mathrm{k}}} \equiv \delta_{L L^{\prime}}-T_{L L}^{\overrightarrow{\mathrm{k}}} \cdot /\left[\omega_{l}\left(D_{l}\right)-\omega_{l}(l)\right]
$$

and

$$
\Omega_{L L^{\prime}}^{\overrightarrow{\mathrm{k}}} \equiv \omega_{l}\left(D_{L}\right) \delta_{L L^{\prime}}-\omega_{l}(l) T_{L L^{\prime}}^{\overrightarrow{\mathrm{k}}} /\left[\omega_{l}\left(D_{L}\right)-\omega_{l}(l)\right],
$$

while $A_{L_{j}}^{\overrightarrow{\mathrm{k}}}$ are the LMTO eigenvectors, satisfying the eigenvalue equations (1.1) and the orthonormality conditions

$$
\sum_{L} \sum_{L} A_{L}^{\mathrm{k}^{*}{ }^{\prime}{ }^{\prime}}, O_{L}^{\overrightarrow{\mathrm{k}}{ }^{\prime}{ }_{L}} A_{L j}^{\overrightarrow{\mathrm{k}}}=\delta_{j^{\prime}{ }_{j}} .
$$

\section{Accuracy and the ASA}

The energy-independent MTO is not an exact solution of Schrödinger's equation in the atomic polyhedron; its radial part is $\Phi(-l-1, r)$ rather than $\phi(E, r)$ inside the MT sphere and its kinetic energy is zero rather than $E-V_{\text {mtz }}$ in the interstitial region. We shall now investigate the induced errors which, for the energy bands, are of order $\left(E-E_{\nu}\right)^{4}$ and $\left(E-V_{\mathrm{mtz}}\right)^{2} \Omega_{I}^{2}$, respectively. The errors of the eigenvectors are of order $\left(E-E_{\nu}\right)^{4}$ and $\left(E-V_{\mathrm{mtz}}\right) \Omega_{\mathrm{r}}$.

We first consider the effect of using $\Phi(r)$ instead of $\phi(r)$ and therefore neglect the error from the interstitial region, i.e., we assume that $V_{\mathrm{mtz}}=E$ or use the ASA. Since the MTO is continuous and differentiable, the one-center expansion of the Bloch sum of exact MTO' ${ }^{12,14} \chi_{L}^{\overrightarrow{\mathrm{k}}}(E, \overrightarrow{\mathrm{r}})$ is given by (4.7) if we choose $D_{L}=D_{l}(E)$ and then substitute $\Phi(D, \overrightarrow{\mathrm{r}})$ by $[\Phi(D) / \phi(E)] \phi(E, \vec{r})$. The condition that the linear combination

$$
\sum_{L} A_{L} \chi_{L}^{\overrightarrow{\mathrm{k}}}(E, \overrightarrow{\mathbf{r}})
$$

of exact MTOs is a solution of Schrödinger's equation at energy $E$, is then that the terms proportional to $\Phi_{L},\left(l^{\prime}, \overrightarrow{\mathbf{r}}\right)$ in the one-center expansion vanish. ${ }^{12,14}$ From (4.7), this leads to the set of homogeneous, linear equations

$$
\sum_{L} T_{L^{\prime} L}^{\overrightarrow{\mathbf{x}}}\left(D_{l} \cdot(E), D_{l}(E)\right) A_{L}=0,
$$

which only have a nontrivial solution for the specified $E$ and $\overrightarrow{\mathrm{k}}$ if

$$
\left|-2(2 l+1) \frac{D_{l}(E)+l+1}{D_{l}(E)-l} \delta_{L}{ }_{L}+S_{L}^{\overrightarrow{\mathrm{k}}{ }_{L}}\right|=0,
$$

as seen from (4. 8). These are the KKR equations for $V_{\mathrm{mtz}}=E$ or in the ASA, ${ }^{17}$ yielding the exact energy $E$ and eigenvector $A_{L}$. We now consider the variational estimates $E_{j}$ and $A_{L j}$, obtained from (1.1) with the LMTO Hamiltonian and overlap matrices given by (4.9) and (4.10). These estimates satisfy

$$
\sum_{L}\left\langle\chi_{L}^{\overrightarrow{\mathbf{k}}} \cdot\left|H-E_{j}\right| \chi_{L}^{\vec{k}}\right\rangle A_{L j}=0,
$$

whereas the substitution of the exact energy and eigenvector into the LMTO secular equations yields

$$
\sum_{L}\left\langle\chi_{L}^{\overrightarrow{\mathrm{k}}} \cdot|H-E| \chi_{L}^{\overrightarrow{\mathrm{k}}}\right\rangle A_{L}=o\left(\left(E-E_{\nu}\right)^{3}\right) A_{L^{\prime}} \cdot
$$

These equations have been obtained by choosing $D_{L}=D_{l}(E)$, writing $T_{L}^{\overrightarrow{\mathrm{k}}}{ }^{\prime}{ }_{L}$ as a factor of the formal two- and three-center integrals, and using (4.22a) together with (2.17) and (2.23), or together with (2.24). Hence, the LMTO energies and eigenvectors are correct to order $\left(E-E_{\nu}\right)^{3}$.

The eigenfunctions (4.21) are only correct to or$\operatorname{der} E-E_{\nu}$, as is expected from the variational principle. However, since the eigenvectors of the LMTO method in the ASA are correct to third order, it is possible to obtain eigenfunctions of the same accuracy. Hence, from the LMTO eigenvalue $E_{j}$ and eigenvector $A_{L j}\left(D_{L}\right)$, corresponding to a normalization specified by some $D_{L}$ 's, we seek the coefficients in the one-center expansion of the wave function correct to order $\left(E-E_{\nu}\right)^{3}$. From the condition of tail cancellation, expressed by (4.22a) and (4.7), we obtain

$$
\begin{aligned}
\Psi_{j} & =\sum_{L} A_{L} \chi_{L}^{\overrightarrow{\mathrm{k}}}(E, \overrightarrow{\mathrm{r}}) \\
& =\sum_{L} A_{L} \Phi_{l}\left(D_{l}(E)\right) \frac{\phi_{L}(E, \overrightarrow{\mathrm{r}})}{\phi_{l}(E)}
\end{aligned}
$$




$$
=\sum_{L} \frac{(2 l+1) \chi_{l}\left(D_{L}\right) A_{L j}\left(D_{L}\right)}{\left[l-D_{l}\left(E_{j}\right)\right] \phi_{l}\left(E_{j}\right)} \phi_{L}\left(E_{j}, \overrightarrow{\mathrm{r}}\right)
$$

to order $\left(E-E_{\nu}\right)^{3}$. In the third line we have substituted $A_{L}$ by $A_{L j}\left(D_{l}(E)\right)$ which then, by the change of normalization (4.5), has been expressed in terms of $A_{L j}\left(D_{L}\right)$. We have further used (4.6) and substituted $E$ by $E_{j}$. For the partial wave $\phi_{L}\left(E_{j}, \overrightarrow{\mathbf{r}}\right)$, we may then use its Taylor series to third order in energy, and the denominator in (4.23) may, to the same order and with (2.18) and (2.25), be written as

$$
\begin{aligned}
{\left[l-D\left(E_{j}\right)\right] \phi\left(E_{j}\right)=} & \left(l-D_{\nu}\right) \phi\left(E_{j}\right) \\
& +\left(\epsilon / S \phi_{\nu}^{2}\right)\left(\phi_{\nu}+\frac{1}{6} \varsigma^{2} \ddot{\phi}_{\nu}\right),
\end{aligned}
$$

where $\epsilon \equiv E_{j}-E_{\nu}$ and $\phi\left(E_{j}\right)$ on the right-hand side should be substituted by its Taylor series to third order. If the LMTO eigenvector is normalized as in (4.21d), the wave function (4.23) will only be normalized to order $E-E_{\nu}$ but since $\left\langle\phi_{L}(E) \mid \phi_{L}(E)\right\rangle$ $=\delta_{L^{\prime} L}$ it may easily be renormalized.

We now estimate the effect on the band structure of using trial functions with improper kinetic energy in the interstitial region. Let $E \equiv E_{j}^{\overrightarrow{\mathrm{k}}}$ and $\Psi \equiv \Psi_{j}^{\overrightarrow{\mathrm{k}}}$ be, respectively, an exact nondegenerate eigenvalue and the corresponding eigenfunction of the MT Hamiltonian $H$, which is $-\nabla^{2}+V_{\mathrm{mtz}}$ in the interstitial region. Correspondingly, $E+d E \equiv E_{j}^{\prime \overrightarrow{\mathrm{k}}}$ and $\Psi+d \Psi$ $\equiv \Psi_{j}^{\prime \vec{k}}$ are the eigenvalue and normalized eigenfunction of the MT Hamiltonian $H+d H$, where $d H$ is small and constant in the interstitial region and vanishes elsewhere. When we then use $\Psi^{\prime}$ as the trial function for estimating the energy $E$, this estimate is in error by

$$
\begin{aligned}
\Delta E & =\left\langle\Psi^{\prime}|H| \Psi^{\prime}\right\rangle-E \\
& =\left\langle\Psi^{\prime}|H-E| \Psi^{\prime}\right\rangle=\langle d \Psi|H-E| d \Psi\rangle \\
& =\operatorname{Re}\left(\left\langle d \Psi \mid \Psi^{\prime}\right\rangle d E-\left\langle d \Psi|d H| \Psi^{\prime}\right\rangle\right) .
\end{aligned}
$$

Since both $\Psi$ and $\Psi^{\prime}$ are normalized, $\operatorname{Re}\left\langle d \Psi \mid \Psi^{\prime}\right\rangle$ $=\frac{1}{2}\langle d \Psi \mid d \Psi\rangle$, moreover, $d E=\langle\Psi|d H| \Psi\rangle+o(d H)$, so that the error may be written, to second order in $d H$, as

$\Delta E=-\operatorname{Re} \frac{\langle d \Psi|d H| \Psi\rangle\langle\Psi|d H| \Psi\rangle}{d E}=-\frac{(d H)^{2}}{4} \frac{d W_{I}^{2}}{d E}$,

where $W_{I} \equiv\left\{\Psi^{2}\right\}-\left\langle\Psi^{2}\right\rangle$ is the probability that the electron is in the interstitial region of volume $\Omega_{I}$. Hence, the error tends to be largest near the centers of wide bands. For the case considered $d H$ $=E-V_{\mathrm{mtz}}$ and, if we assume that only one value of $l$ contributes significantly to the sum (4.23), that $\phi_{l}(E, r)$ is slowly varying in the interstitial region, and that the atomic polyhedron is almost a sphere, we obtain the convenient expression

$$
\Delta E_{l} \approx\left(E-V_{\mathrm{mtz}}\right)^{2}\left[-\dot{\phi}_{l}(E) \phi_{l}^{3}(E)\right]\left(\Omega_{I} / 4 \pi\right)^{2}
$$

relating the error to the potential parameters and the volume of the interstitial region.

There are conceptual advantages of using everywhere the atomic sphere rather than the less-welldefined MT sphere. In practice, we therefore always take $S=(3 \Omega / 4 \pi)^{1 / 3}$, in the case of one atom per primitive cell, and we compute the potential parameters from integrals in the atomic sphere, or from logarithmic derivative functions at the atomic sphere, using a potential which is the spherical MT potential inside the MT sphere and the constant $V_{\mathrm{mtz}}$ between the MT and atomic spheres. Now, the combined correction term in (4.12) and (4.13) merely corrects for the departure of the atomic polyhedron from the atomic sphere of the same volume and for the neglect of the higher partial waves. As a consequence, the energy bands will depend on the potential through the potential parameters at the atomic sphere and only slightly on $V_{\mathrm{mtz}}$ through (4.12).

The expansion (4.4) converges inside the atomic sphere at the origin but, when we use the one-center expansion (4.3) or (4.7) for the atomic-sphere orbitals, we treat any nearest-neighbor orbital incorrectly in the region where its own atomic sphere overlaps the atomic sphere at the origin. The orbital is, however, continuous and differentiable and the error amounts to using a curvature corresponding to the kinetic-energy zero instead of $E-V_{\mathrm{mtz}}$. For the orbital at the origin, on the other hand, we use the correct kinetic energy, rather than zero, in the region between the inscribed and atomic spheres at the origin. As a result, (4.24) seems to describe the error, which is quadratic in $\Omega_{I} d H$, quite well when we let $\Omega_{T}$ be the volume between the atomic polyhedron and its inscribed sphere.

In Fig. 5, we show the errors for the unfavorable case of wide bands, specifically the free-electron bands computed in an hcp crystal. The exact bands and those computed with the LMTO method are shown in, respectively, the first and the last panels of Fig. 6. The points in Fig. 5 indicate the errors of the energies at all the high-symmetry points computed with the LMTO method using (4.12) and (4.13) with $S$ being the atomic-sphere radius and with potential parameters obtained from the formulas of Sec. II D. Two different values of $E_{\nu}$ were used to cover the range of energies shown in order that the fourth-order error from the spherical region could be neglected. The curves are the results of (4.24) and the good agreement confirms the relevance of this expression, also when the atomic radius is used. The one exception is for the $d f-$ like $K_{2}$ level at $E S^{2}=9.88$, and its additional error is simply due to our neglect of the $f$ orbitals in the present calculation. For free electrons, the Fermi level corresponding to an occupancy of $n$ electrons per atom is

$$
\tilde{E}_{F} S^{2}=\left(\frac{9}{4} \pi n\right)^{2 / 3}=3.68 n^{2 / 3},
$$




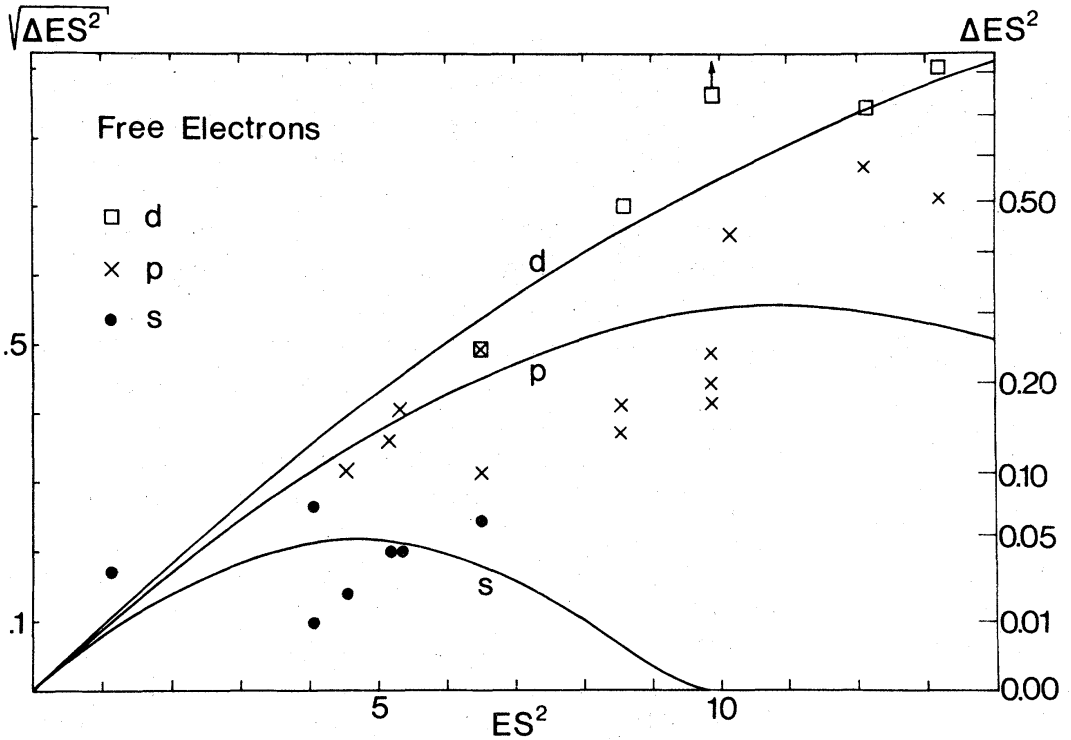

FIG. 5. Errors of the free-electron eigenvalues, computed with the LMTO method using (4.12) and (4.13) with $l \leq 2$, at the symmetry points $\Gamma, K$, $M, H, L$, and $A$ of an hep crystal. Points, crosses, and squares denote $s-, p-$, and $d$-like states, respectively, and curves show the $s, p_{2}$ and $d$ estimates $(4.24)$. The atomic rather than the MT sphere was used throughout, and the errors proportional to $\left(E-E_{\nu}\right)^{4}$ were suppressed by using two different values of $E_{\nu}$. and it is indicated for $n=1,2$, and 3 on the righthand scale of Fig. 6. We conclude that the LMTO method can yield the occupied bands to within a few percent of the Fermi energy for all metals. For narrow bands, like the $d$ bands of transition metals, the errors are negligible.

In summary, the error from the interstitial region is usually larger than the error from the spherical region and, whereas the latter error is arbitrary in the sense that $E_{\nu}$ is arbitrary, we cannot choose any other kinetic energy than zero in the interstitial region ${ }^{12}$ without giving up the important concept of canonical bands and structure constants that we shall describe in Sec. IV D.

Finally, we define the ASA as the approximation of using the Eqs. (4.22) with the logarithmic derivative functions and the structure constants appropriate for the atomic sphere and truncating the secular matrix at $l=2$ or 3 . The Hamiltonian and overlap matrices of the corresponding LMTO method, correct to third order in $E-E_{\nu}$, are then given by (4.9) and (4.10). The eigenvalues of the ASA have small errors of order $E-V_{\mathrm{mtz}}$ in addition to the second-order errors (4.24), and they are exhibited

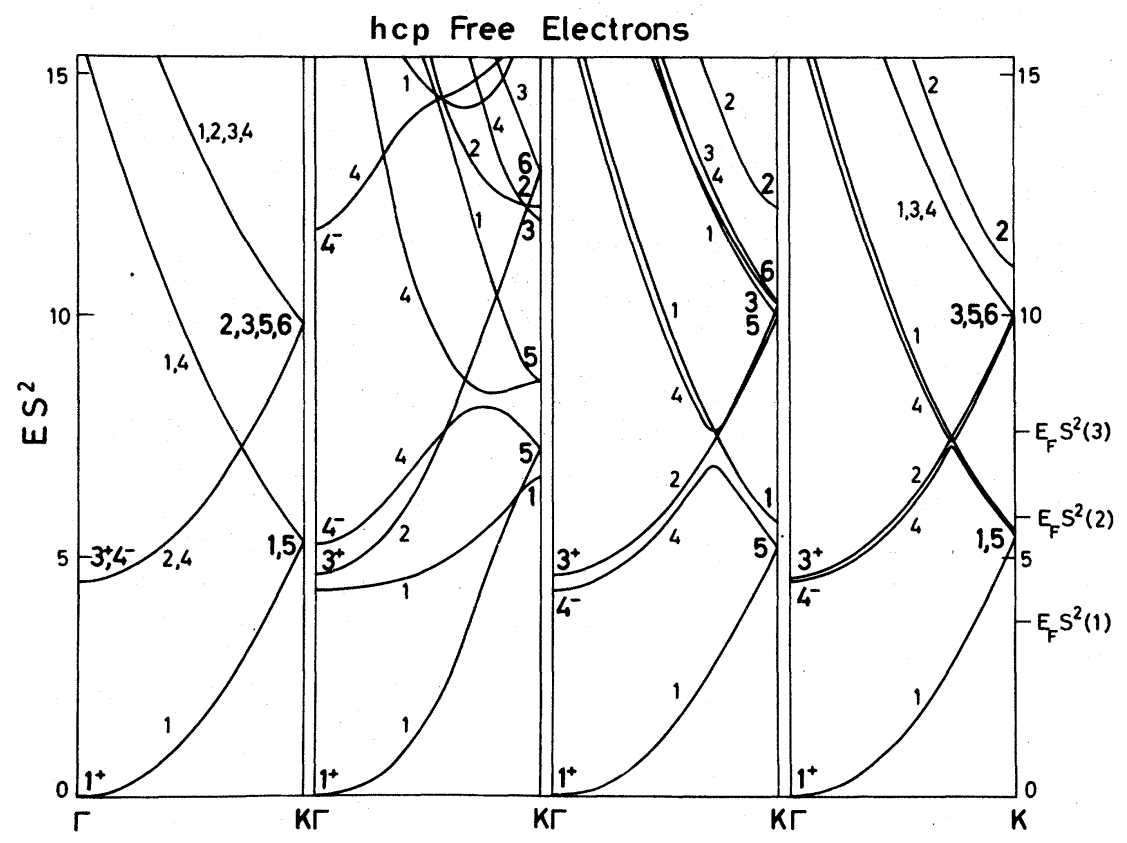

FIG. 6. Free-electron bands in the hcp structure. Panels from left to right show: (1) Exact bands. (2) Pure bands in the ASA (4.36). From the bottom, $T_{1}$ and $T_{4}$ are the two first $s$ bands, $T_{1}, T_{2}, T_{4}$, $T_{1}, T_{4}$, and $T_{3}$ are the six first $p$ bands, and $T_{4}$, $T_{2}$, and $T_{1}$ are the lowest of the ten first $d$ bands. (3) Hybridized bands in the ASA, (4.9) and (4.10) with $l \leq 2$. (4) LMTO bands, (4.12) and (4.13) with $l \leq 2$. The errors of the levels at $\Gamma$ and $K$ are shown in Fig. 5. On the right-hand scale are shown the Fermi levels for occupancies of 1-3 electrons per atom. 
by the free-electron bands in the ASA shown in the third panel of Fig. 6. Whereas the ASA may not be sufficiently accurate for the most detailed Fermi surface calculations, it is highly efficient in generating, through self-consistent calculations employing (4.23), the spherical average of the electron distribution in each sphere.

\section{Canonical bands and structure constants}

We shall now investigate the properties of the structure constants $S_{L}^{\overrightarrow{\mathrm{k}}}{ }_{L}$ defined in (4.4). In the ASA, this matrix contains all information about the arrangement of the atoms, and we shall show that it is canonical in the sense that it depends neither on the energy, the potential, nor the atomic volume. Hence, in the ASA, the band-structure problem is reduced to that of finding the eigenvalues and eigenfunctions for a single atomic sphere, subject to the anisotropic and $\overrightarrow{\mathrm{k}}$-dependent boundary condition specified by $S_{L}^{\overrightarrow{\mathrm{k}}}{ }_{L}$ and expressed by (4.22). Of particular significance are the eigenvalues $S_{l i}^{\vec{k}}$ of the $l l$ subblocks of the structure matrix $s_{L}^{\vec{k}}{ }_{L}$ and we have named them canonical bands.

From the expansion (4.4) of the $2^{l}$-pole field, the structure constants in the $l m$ representation are given explicitly as

$$
\mathrm{S}_{l^{\prime} m^{\prime} ; l m}^{\overrightarrow{\mathrm{k}}}=g_{l^{\prime} m^{\prime} ; l m} \sum_{l^{\circ}+l, m^{\circ}-m}^{\overrightarrow{\mathrm{s}}}
$$

with

$$
\sum_{\lambda \mu}^{\overrightarrow{\mathrm{k}}} \equiv \sum_{\overrightarrow{\mathrm{R}} \neq \overrightarrow{0}} e^{i \overrightarrow{\mathrm{k}} \cdot \overrightarrow{\mathrm{R}}}\left(\frac{S}{R}\right)^{\lambda+1}\left[(\sqrt{4} \pi) i^{\lambda} Y_{\lambda \mu}(\hat{R})\right]^{*}
$$

and

$$
\begin{aligned}
g_{l^{\prime} m^{\prime} ; l m} & \equiv \frac{-2(2 \lambda-1) ! !}{\left(2 l^{\prime}-1\right) ! !(2 l-1) ! !}(2 \lambda+1)^{1 / 2} c^{\lambda}\left(l^{\prime} m^{\prime} ; l m\right) \\
& =(-)^{m+1} 2\left(\frac{\left(2 l^{\prime}+1\right)(2 l+1)}{2 \lambda+1} \frac{(\lambda+\mu) !(\lambda-\mu) !}{\left(l^{\prime}+m^{\prime}\right) !\left(l^{\prime}-m^{\prime}\right) !(l+m) !(l-m) !}\right)^{1 / 2},
\end{aligned}
$$

where $\lambda \equiv l^{\prime}+l$ and $\mu \equiv m^{\prime}-m$. Moreover, $(-1) ! ! \equiv 1$ and

$$
\begin{aligned}
c^{\lambda}\left(l^{\prime} m^{\prime} ; l m\right) & \equiv[4 \pi /(2 \lambda+1)]^{1 / 2} \int d \hat{r} Y_{\lambda \mu}(\hat{r}) Y_{l^{\prime} m^{\prime}}^{*}(\hat{r}) Y_{l m}(\hat{r}), \\
& =\left(\frac{2 l+1}{2 l^{\prime}+1}\right)^{1 / 2} A_{000}^{\lambda l t^{\prime}} A_{\mu m m^{\prime}}^{\lambda l l^{\prime}}
\end{aligned}
$$

are the Gaunt coefficients, while $A$ are the Wigner or Clebsch-Gordan coefficients. Our structure constants may be expressed in terms of the original KKR structure constants ${ }^{6}$

$$
\begin{aligned}
\otimes_{\frac{\overrightarrow{\mathbf{r}}}{l^{\prime} m^{\prime} ; l m}}(\kappa)= & \sum_{\lambda}(2 \lambda+1)^{1 / 2} c^{\lambda}\left(l^{\prime} m^{\prime} ; l m\right) \\
& \times \sum_{\overrightarrow{\mathrm{R}} \neq \overrightarrow{0}} e^{i \overrightarrow{\mathbf{k}} \cdot \overrightarrow{\mathrm{R}}} \kappa n_{\lambda}(\kappa R)\left[(\sqrt{4} \pi) i^{\lambda} Y_{\lambda \mu}(\hat{R})\right] *,
\end{aligned}
$$

where $n_{\lambda}$ are the spherical Neumann functions, as

$$
S_{l^{\prime} m^{\prime} ; l m}^{\vec{k}}=\lim _{\kappa^{2} \rightarrow 0} \frac{Q_{l^{\prime} m^{\prime} ; l m}^{\vec{k}}(\kappa)}{\left(\sqrt{\frac{1}{2}} S\right) \kappa n_{l} \cdot(\kappa S)\left(\sqrt{\left.\frac{1}{2} S\right) \kappa n_{l}(\kappa S)}\right.},
$$

and they have a number of simple properties.

First of all, they are independent of energy $E$ $=\kappa^{2}+V_{\mathrm{mtz}}$ and, by specifically choosing $\kappa^{2}=0$, we achieve that they are also invariant under uniform scaling of the lattice. Second, the poles $\left(|\vec{k}+\vec{G}|^{2}\right.$ $\left.-\kappa^{2}\right)^{-1}$ of the KKR structure constants have been reduced to singularities at the reciprocal-lattice points only. Specifically,

$$
\lim _{k \rightarrow 0} \sum_{\lambda \mu}^{\overrightarrow{\mathrm{k}}} \frac{3(k S)^{\lambda}(\sqrt{4} \pi) Y_{\lambda \mu}^{*}(\hat{k})}{(2 \lambda-1) ! !(k S)^{2}}+\text { const }
$$

so that only the $s s$ and $s p$ structure constants diverge while the $p p$ structure constants are discontinuous at the center of the Brillouin zone. Third, there is no sum on $\lambda$ in (4.25), the Gaunt coefficients are particularly simple when $\lambda=l^{\prime}+l$, and the spherical Bessel and Neumann functions, entering the KKR formalism, become $r^{l}$ and $r^{-l-1}$, respectively. Our structure constants are Hermitian and, in the present case of one atom per primitive cell and with $\left(l^{\prime}, l\right) \leq 2$, only the 15 complex lattice summations (4.26) with $\lambda \leq 4$ and $0 \leq \dot{\mu} \leq \lambda$ must be performed. This may be done by the standard Ewald technique although, for the five contributions to the $d d$ block, the convergence of (4.26) is so fast that this technique is not necessary. In any case, the structure constants need only be computed once and for all throughout the irreducible part of the Brillouin zone of each crystal structure.

When including the correction of the ASA, an extra set of structure constants, namely, $\mathfrak{F}_{l^{\prime}}^{\mathbf{l}} m^{\prime} ; l m$ in (4.17) are needed. These constants, too, are independent of energy and volume and must only be computed once for each structure.

The MT and atomic-sphere approximations take advantage of the fact that, although periodic, the 
crystal potential is spherically symmetric in the neighborhood of each atom. Our potential parameters do not therefore depend on the quantum number $m$, and the only coupling between the $m$ 's of a given $l$ is provided by the long-range asphericity of the crystal potential, that is, by the structure constants. It is then convenient to use an angular momentum representation in which the $l l$ subblocks of the structure constants are diagonal. Hence, if $S_{l i}^{\overrightarrow{\mathbf{k}}}$ are the eigenvalues and $U_{l m ; l i}^{\overrightarrow{\mathbf{k}}}$ the eigenvectors of $\dot{s}_{l m^{\prime} ; l m}^{\vec{k}}$

$$
\begin{aligned}
& \sum_{l^{\prime} m^{\prime}} \sum_{l m} U_{l^{\prime} m^{\prime} ; l^{\prime} i^{\prime}}^{\overrightarrow{\vec{k}} \delta_{l^{\prime} m^{\prime} ; l m}^{\overrightarrow{\mathrm{k}}}} U_{l m ; l i}^{\overrightarrow{\mathrm{k}}} \\
& =\delta_{l^{\prime}, l \delta_{i^{\prime} i}} S_{l i}^{\overrightarrow{\mathrm{r}}_{i}^{*}}+\left(1-\delta_{l^{\prime}, l}\right) \delta_{l^{\prime} i^{\prime} ; l i}^{\overrightarrow{\mathrm{k}}} \text {. }
\end{aligned}
$$

In Sec. IV $\mathrm{E}$ we shall show that the unhybridized $n l$ energy band may be defined as the canonical $l$ band $S_{l i}^{\overrightarrow{\mathbf{k}}}$ subject to a nonuniform change of scale, $S \rightarrow E$, and the similarity of for instance the hcp canonical $d$ band structure with the energy-band structure of the transition metal os may be appreciated when comparing Figs. 2 and 4 in Paper II. The canonical $l$ band consists of $2 l+1$ subbands numbered by the index $i$ and, for $l>0$, the center of gravity is zero for each value of $\vec{k}$, i.e.,

$$
\begin{aligned}
\sum_{i=1}^{2 l+1} S_{l i}^{\overrightarrow{\mathbf{k}}} & =\sum_{m=-l}^{l} \delta_{l m ; l m}^{\overrightarrow{\mathrm{k}}} \\
& \propto \sum_{m} c^{2 l}(l m ; l m) \propto \int d \hat{r} Y_{2 l, 0}(\hat{r})=0
\end{aligned}
$$

for $l>0$. This follows from the invariance of the trace to unitary transformations, from (4.25) and (4.28a), and from Unsöld's theorem, ${ }^{21}$ stating that

$$
\sum_{m}\left|Y_{l m}(\hat{r})\right|^{2}
$$

is independent of $\hat{r}$. For all canonical bands, including the $s$ band, the first moment vanishes

$$
\frac{1}{2 l+1} \int_{0}^{2 l+1} \delta_{l}(n) d n \equiv \frac{1}{2 l+1} \sum_{i=1}^{2 l+1} \frac{\Omega}{8 \pi^{3}} \int_{\mathrm{BZ}} \delta_{l i}^{\overrightarrow{\mathrm{k}}} d^{3} k=0
$$

as follows from (4.26). We have defined the canonical function $s_{l}(n)$, which is "the Fermi energy on the $s$ scale corresponding to an occupancy of $n$ states per atom in the canonical $l$ band," and the density of states in the canonical band is

$$
\Re_{l}(s)=1 / S_{l}^{\prime}(n) .
$$

The expression for the second moment is also very simple. From (4.25), (4.26), (4.28b), the unitarity of the Wigner coefficients, and Unsöld's theorem, we obtain

$\frac{1}{2 l+1} \int_{0}^{2 l+1} \delta_{l}^{2}(n) d n=2^{l+2}(2 l+1)$

$$
\times \frac{(2 l+1)(2 l+3) \cdots(4 l-1)}{1 \times 2 \cdots \times l} \sum_{\overrightarrow{\mathrm{R}} \neq \overrightarrow{0}}\left(\frac{S}{R}\right)^{2(2 l+1)},
$$

which only depends on the number of atoms in the various shells of the lattice and the distance between the shells. For structures with several equivalent atoms per primitive cell, these relations for the first and second moments still hold, provided that $\vec{R}$ in (4.34) runs over the positions of all equivalent atoms measured from a particular one.

The second moment of the $s$-canonical band diverges owing to the singularity at the center of the zone. The second moments of the $p$-canonical bands of the bcc, fcc, and hcp $\left(c / a=\sqrt{\frac{8}{3}}\right)$ structures are, respectively, 29.4, 29.2, and 28.8 which, for an idealized, rectangular shape of the state density, correspond to the respective bandwidths $(12 \times 29.4)^{1 / 2}=18.8,18.7$, and 18.6. The similarly defined canonical $d$ band widths are 23. 8, 23.5, and 23.5 for the bcc, fcc, and hcp structures, respectively. Hence, the canonical bandwidths of the three structures are essentially the same and the magnitudes are in close agreement with the empirical Wigner-Seitz rule, which states that a band of primarily $l$ character extends over the range of energies where the corresponding logarithmic derivative is negative. From (4.22), and with the neglect of $l^{\prime} l$ hybridization, that is, from (4.36) below, this rule may be expressed as

$$
-2(2 l+1)\left(1+l^{-1}\right) \leq \mathcal{S}_{l i}^{\overrightarrow{\mathrm{k}}} \leqslant 2(2 l+1),
$$

and it yields canonical $p$ and $d$ bandwidths of, respectively, 18 and 25. As seen from (4.29), this rule is exact at the bottom of the $s$ band, and it holds within $10 \%$ for the extrema of all the canonical bands in closely packed monoatomic solids that we have so far calculated. ${ }^{16}$

\section{E. Pure bands and hybridization}

We now define the pure or unhybridized energyband structure as it is given by the ASA (4.22) when neglecting the off-diagonal blocks of the structure constants. The pure $n l$ band is therefore the $n$th solution of

$$
2(2 l+1) \frac{D_{l}(E)+l+1}{D_{l}(E)-l}=\mathrm{s}_{l i}^{\overrightarrow{\mathrm{k}}},
$$

where the potential function on the left-hand side, according to (2.31), increases monotonically between its asymptotes, and where the canonical $l$ band, according to (4.34), is bounded for $l>0$ and is upwards bounded for $l=0$. As a consequence, the relation (4.36) between the canonical $l$ band and the pure $n l$ bands are $\overrightarrow{\mathrm{k}}$-independent scalings, specified by the $l$-logarithmic derivative function. From the one-center expansion leading to (4.22), it may 
be realized that a pure $l$ band is defined by the property that the Bloch sum of exact MTO's $\chi_{l i}^{\overrightarrow{\mathbf{k}}}(E, \overrightarrow{\mathrm{r}})$ is the exact solution in all atomic spheres of the radial Schrodinger equation for the $l$ th partial wave.

For the Bloch sum of energy-independent MTO's it is also convenient to use the $l i$ representation, and in this case we specify the normalization and arrangement of terms in the LMTO matrices by choosing $D_{L}=D_{l i}^{\overrightarrow{\mathrm{k}}}$, where

$$
D_{l i}^{\overrightarrow{\mathrm{k}}_{i}} \equiv-l-1-(2 l+1) \mathrm{s}_{{ }_{i}}^{\overrightarrow{\mathrm{k}}_{i}} /\left[2(2 l+1)-\mathrm{s}_{l_{i}}^{\overrightarrow{\mathrm{k}}}\right],
$$

so that the diagonal blocks of $T_{l^{\prime}}^{\overrightarrow{\mathrm{k}}} i^{\prime} ; l_{i}$ in (4.8) vanish. The Bloch sum $\chi_{l i}^{\overrightarrow{\mathrm{k}}}\left(D_{l i}^{\overrightarrow{\mathrm{k}}}, \overrightarrow{\mathrm{r}}\right)$ only has one angular momentum component of the same $l$, namely, $\Phi_{l i}\left(D_{l i}^{\overrightarrow{\mathbf{k}}}, \overrightarrow{\mathrm{r}}\right)$, in its one-center expansion (4.7), and the corresponding formal one-center energy is the variational estimate $E_{l}\left(D_{l i}^{\overrightarrow{\mathbf{k}}}\right)(2.17)$ of the pure $\nu l$ energy band given implicitly in (4.36). From (2. 12),

$$
\begin{aligned}
\omega_{l}\left(D_{l i}^{\overrightarrow{\mathbf{k}}_{i}}\right)= & \omega_{l}(-l-1) \\
& +\frac{1}{2} S \Phi_{l}^{2}(-l-1) \mathrm{s}_{l i}^{\overrightarrow{\mathbf{k}}} /\left(1-\gamma_{l} \mathrm{~s}_{l i}^{\overrightarrow{\mathbf{k}}}\right),
\end{aligned}
$$

where

$$
\begin{aligned}
\gamma & \equiv \frac{1}{2(2 l+1)} \frac{\Phi(-l-1)}{\Phi(l)}, \\
& =\frac{\omega(-l-1)-\omega(l)}{2(2 l+1)^{2} S \Phi^{2}(l)}=\frac{\frac{1}{2} S \Phi^{2}(-l-1)}{\omega(-l-1)-\omega(l)},
\end{aligned}
$$

and the alternative expressions follow from (2.15).

We may define the energies $C_{\nu l}$ and $V_{\nu l}$ as those where the $l$-logarithmic derivative function takes the values $-l-1$ and $l$, respectively. For free electrons, these energies were specifically considered in Sec. IID and since $\tilde{V}_{0 l}=0$ we name $V_{\nu l}$ $\approx E_{l}(l)$ the square-well pseudopotential. From (4. 31) and (4.36) we name $C_{\nu l} \approx E_{l}(-l-1)$ the center of the band. We further define the intrinsic band masses

$$
\begin{aligned}
\mu_{\nu l} & \equiv\left[\frac{1}{2} S^{3} \phi_{l}^{2}\left(C_{\nu l}\right)\right]^{-1} \\
& =-2 S^{-2} \dot{D}_{l}\left(C_{\nu l}\right) \approx-2\left[S^{2} E_{l}^{\prime}(-l-1)\right]^{-1} \\
& =\left[\frac{1}{2} S^{3} \Phi^{2}(-l-1)\right]^{-1} \frac{\left[1+\left\langle\dot{\phi}_{\nu}^{2}\right\rangle \omega^{2}(-l-1)\right]^{2}}{1-\left\langle\dot{\phi}_{\nu}^{2}\right\rangle \omega^{2}(-l-1)}
\end{aligned}
$$

and

$$
\tau_{\nu l} \equiv\left\{[1 /(2 l+3)] S^{3} \phi_{l}^{2}\left(V_{\nu l}\right)\right\}^{-1},
$$

which, from the values of the free-electron probability density $\tilde{\phi}_{l}^{2}$ given in Sec. IID, are seen to equal, respectively, $\mu(-l-1)$ and $\mu(l)$, as defined in (3.7). If we then put $E_{\nu}=C_{\nu l}$ in (2.17) and (4.38), we may state that the pure $\nu l$ energy-band structure is derived from the canonical $l$ band structure by fixing the band position through $C_{\nu l}$, scaling it by $\mu_{\nu l} S^{2}$, and distorting it nonlinearly by $\Phi_{l}(-l-1) /$ $\Phi_{l}(l)$ and $\left\langle\dot{\phi}_{\nu l}^{2}\right\rangle$. The potential parameters for hcp transition metals may be found in Tables I and II of Paper II, and those for free electrons were given in Table I. For a given $l$, the values of the distortion parameters $\Phi(-l-1) / \Phi(l)$ and $\left\langle\dot{\phi}_{\nu}^{2}\right\rangle$ vary little among similar atoms provided that the $D_{\nu}$ 's are similar and, using typical values, we find from (4. 35) and (4.38) that the width of the pure band is of order $10 S \Phi^{2}(-l-1)$. For transition-metal $d$ bands $\Phi(-3) / \Phi(2)$ is numerically far less than unity, the distortion is therefore small and the $d$ band extends approximately from $7.5 S \Phi^{2}(-3)$ below to $5 S \Phi^{2}(-3)$ above the band center. For the bottom of the $s$ band, we find from (4.25), (4.27), and (4.29) that $s_{00}^{\mathbf{k}} \rightarrow-6(k S)^{-2}$, and with $E_{\nu}=V_{s},(4.38)$, (4. 39), and (4.41),

$$
\lim _{k \rightarrow 0} E_{s}\left(D_{s}^{\overrightarrow{\mathbf{k}}}\right)=V_{s}+k^{2} / \tau_{s},
$$

as expected. The pure bands for hcp free electrons are shown in the second panel of Fig. 6.

The hybridization matrix in the $l i$ representation is

$$
\begin{aligned}
T_{l^{\prime} i^{\prime} ; l i}^{\vec{k}}\left(D_{l^{\prime} i^{\prime}}^{\overrightarrow{\mathbf{k}}} ; D_{l i}^{\overrightarrow{\mathbf{k}}}\right)= & \frac{\left(\sqrt{\frac{1}{2}} S\right) \Phi_{l^{\prime}}\left(-l^{\prime}-1\right)}{1-\gamma_{l^{\prime}} \delta_{l^{\prime}}^{\vec{k}} i^{\prime}} S_{l^{\prime} i^{\prime} ; l i}^{\overrightarrow{\mathrm{k}}} \\
& \times \frac{\left(\sqrt{\frac{1}{2}} S\right) \Phi_{l}(-l-1)}{1-\gamma_{l} \delta_{l i}^{\overrightarrow{\mathbf{k}}}}
\end{aligned}
$$

which vanishes for $l^{\prime}=l$. The Hamiltonian and overlap matrices in the ASA, (4.9) and (4.10), only have contributions to their diagonal elements $\left(l^{\prime}\right.$ $=l$ and $i^{\prime}=i$ ) from the formal one- and three-center integrals. The one-center integrals yield the pureband $E_{l}\left(D_{l i}^{\overrightarrow{\mathbf{k}}}\right)$, which only depends on the potential parameters of the same $l$, while the three-center integrals, with reference to (4.22), may be regarded as the second-order contribution from the partial waves of other angular momenta. The single-LMTO estimate $\left\langle\chi_{l i}^{\overrightarrow{\mathbf{k}}}|H| \chi_{l i}^{\overrightarrow{\mathbf{k}}}\right\rangle /\left\langle\chi_{l i}^{\overrightarrow{\mathbf{k}}} \mid \chi_{l i}^{\overrightarrow{\mathbf{k}}}\right\rangle$ is therefore different from, and more accurate than, the pure-band estimate but it depends on the potential parameters of all angular momenta.

We named $T_{l^{\prime}}^{\overrightarrow{\mathrm{k}}} i^{\prime} ; l i$ the hybridization matrix because the secular equation in the ASA (4.22) may be written as

$$
\begin{aligned}
& 0=\left|\left[\omega_{l}\left(D_{l i}^{\overrightarrow{\mathbf{k}}}\right)-\omega_{l}\left(D_{l}(E)\right)\right] \frac{\omega_{l}\left(D_{l i}^{\overrightarrow{\mathbf{k}}}\right)-\omega_{l}(l)}{\omega_{l}\left(D_{l}(E)\right)-\omega_{l}(l)} \delta_{l^{\prime} l^{\prime} \delta_{i^{\prime}} i+T^{\mathbf{k}^{\prime}}{ }^{\prime} i^{\prime} ; l i}\right|
\end{aligned}
$$

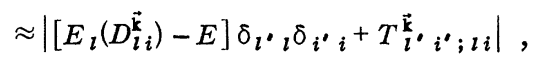


where the simple eigenvalue equation (4.44) is valid when $E \approx E_{l}\left(D_{l i}^{\overrightarrow{\mathbf{k}}}\right)=E_{l^{\prime}}\left(D_{l}^{\overrightarrow{\mathbf{k}}}, i^{\prime}\right)$, and when we neglect $\left\langle\dot{\phi}_{\nu l^{\prime}}^{2}\right\rangle$ and $\left\langle\dot{\phi}_{\nu l}^{2}\right\rangle$, that is, when $E_{\nu}$ is chosen near the assumed crossover of the two pure bands. Under the same conditions, $T_{l^{\prime}}^{\overrightarrow{\mathrm{k}}} i^{\prime} ; i_{i}$ equals the formal two-center contribution to the LMTO hybridization matrix $\left\langle\chi_{\vec{l}}^{\overrightarrow{\mathbf{k}}}, i_{i},|H-E| \chi_{l i}^{\overrightarrow{\mathbf{k}}}\right\rangle$.

The hybridization between the $s$ band and a $d$ band of negligible distortion $\Phi(-3) / \Phi(2)$ is, according to (4.43) and (4.6), described by

$$
T_{s ; d i}^{\overrightarrow{\mathbf{k}}}=\left[\omega_{s}\left(D_{s}^{\overrightarrow{\mathbf{k}}}\right)-\omega_{s}(0)\right] \frac{\Phi_{d}(-3)}{2 \Phi_{s}(0)} s_{s ; d i}^{\overrightarrow{\mathbf{k}}} \rightarrow \frac{-k^{2}}{\left(0.3 \tau_{s} \mu_{d}\right)^{1 / 2}}
$$

for $k \rightarrow 0$. The limiting value is identical with the result of Heine ${ }^{7}$ when the mass at the bottom of the $s$ band is unity, and it has been obtained here by neglecting the parameters $\left\langle\dot{\phi}_{\nu}^{2}\right\rangle$ and using (4.29) for $\boldsymbol{S}_{00 ; 20}^{\overrightarrow{\mathbf{k}}}$ under the assumption that the constant term vanishes as is the case in cubic crystals.

As mentioned in connection with (4.29), the canonical and the pure $p$ band have a branch which is discontinuous at the center of the zone (this is the second $T_{1}$ band in Fig. 6), and the energy band only becomes continuous through hybridization with the $s$ band. In cubic structures the $p$ level at the center of the zone $\Gamma$ is triply degenerate, and when $\overrightarrow{\mathrm{k}}$ approaches $\Gamma$ along the $\hat{z}[001]$ direction, the canonical doubly degenerate $\Delta_{5}$ band $s_{1 \pm 1 ; 1 \pm 1}^{\vec{k}}$ goes continuously to the value 6 but the canonical $\Delta_{1}$ band $s_{10 ; 10}^{\vec{k}}$ tends to -12 . The $\Delta_{5}$ band does not hybridize with the $s$ band while the $s p$ hybridization constant for the $\Delta_{1}$ bands tends to $-6 \sqrt{3}(k S)^{-1}$. For small nonzero $k$ values, (4.22) now yields

$$
\left[1+\frac{1}{6}(k S)^{2} s_{s}(E)\right]\left[s_{p}(E)+12\right]=18,
$$

where

$$
\delta_{l}(E) \equiv 2(2 l+1)\left[D_{l}(E)+l+1\right] /\left[D_{l}(E)-l\right] .
$$

Consequently, the $p$-like solution tends to $18-12$
$=6$ and is therefore continuous at $\Gamma$, regardless of the $s$ potential parameters. The $s$-like solution may still be written in the form of (4.42), but with the mass substituted by

$$
\tau_{s p}=\tau_{s} / D_{p}\left(V_{s}\right) \approx \tau_{s}\left(1-\frac{1}{5} \tau_{p} V_{p s}\right),
$$

where $V_{p s} \equiv\left(V_{p}-V_{s}\right) S^{2}$. This expression is in accord with a result of Bardeen. ${ }^{15}$ The hybridized hcp free-electron energy bands in the ASA are shown in the third panel of Fig. 6.

\section{F. Several atoms per primitive cell}

We shall briefly indicate how the LMTO formalism is modified when the primitive cell contains $h$ spheres, centered at positions $\vec{Q}=\vec{Q}_{t q}$ with associated potentials $v_{t}(|\overrightarrow{\mathrm{r}}-\overrightarrow{\mathrm{Q}}|)$. Groups of identical atoms at equivalent positions are labeled by $t$ while the $h_{t}$ atoms within a group are labeled by $q$. In the case of compounds, there is little justification for using a common interstitial potential $V_{\mathrm{mtz}}$ and in that case we circumvent the MT construction by using the ASA with judiciously chosen sphere radii $S_{t}$. Subject to the condition that the sum of the sphere volumes equals that of the primitive cell, the spheres should according to (4.24) have little overlap and, in the region of overlap, the kinetic energy should be small. The radii specify the regions in which the self-consistently obtained charge density will be spherically symmetric and, subject to the restrictions set by the ASA, they should therefore in principle ${ }^{2}$ be chosen so as to minimize the total energy of the ground state.

In analogy with (4.2) and (4.3), the Bloch sum of MTO's in the tlqm representation is

$$
\chi_{t l \ell m}^{\overrightarrow{\mathrm{r}}}(\overrightarrow{\mathrm{r}})=\sum_{\overrightarrow{\mathrm{R}}} e^{i \overrightarrow{\mathrm{r}} \circ \overrightarrow{\mathrm{R}}} \chi_{t l m}(\overrightarrow{\mathrm{r}}-\overrightarrow{\mathrm{R}}-\overrightarrow{\mathrm{Q}}),
$$

and its expansion in the sphere at $\vec{Q}^{\prime} \equiv \vec{Q}_{t^{\prime} q^{\prime}}$ is

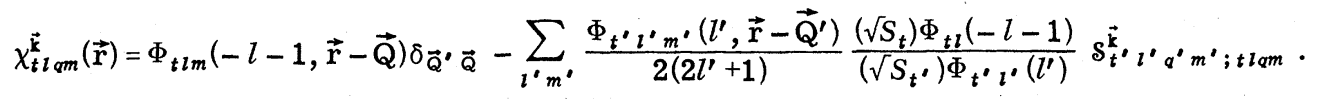

The Hermitian, canonical structure matrix is then

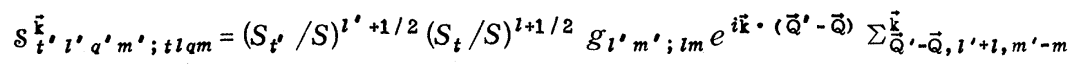

where $S$ is the average radius, defined by the condition that $(4 \pi / 3) S^{3} h=\Omega$. The coefficients $g$ were defined in (4.27) and the lattice sums, periodic in $\vec{\delta} \equiv \vec{Q}^{\prime}-\vec{Q}$, are

$$
\sum \underset{\vec{b} \lambda \mu \mu}{\overrightarrow{\mathbf{k}}} \equiv \sum_{\vec{\Delta} \neq \overrightarrow{0}} e^{i \overrightarrow{\mathbf{k}} \cdot \vec{\Delta}}\left(\frac{S}{\Delta}\right)^{\lambda+1}\left[(\sqrt{4} \pi) i^{\lambda} Y_{\lambda \mu}(\hat{\Delta})\right]^{*}
$$

with $\vec{\Delta} \equiv \vec{R}-\vec{\delta}$. In (4.14) and (4.17), the Fourier transform of the pseudo-MTO is given by

$$
\begin{aligned}
F_{t l q m}(\overrightarrow{\mathrm{K}}) \equiv & (2 l+1)(2 l+3) \\
& \times\left(4 \pi S_{t}^{3} / \Omega\right)\left[j_{l+1}\left(K S_{t}\right) /\left(K S_{t}\right)^{3}\right] Y_{l m}(\hat{K}) e^{-i \overrightarrow{\mathrm{K}} \cdot \overrightarrow{\mathrm{Q}}} .
\end{aligned}
$$

With the following definitions and substitutions, 
the remainder of the LMTO formalism in the previous sections can be taken over directly. If we define the radial functions $\Phi_{t l}(r)$ and $\phi_{t l}(r)$ such that they vanish for $r>S_{t}$, we may use the following generalization of (2.3) and (4.1):

$$
\Phi_{L}(\overrightarrow{\mathrm{r}})=\sum_{q=1}^{h_{t}} \sum_{m=-l}^{l} \Phi_{t l}(|\overrightarrow{\mathrm{r}}-\overrightarrow{\mathrm{Q}}|) i^{l} Y_{l m}(\overrightarrow{\mathrm{r}}=\overrightarrow{\mathrm{Q}}) U_{t l_{q m} ; L}^{*},
$$

and analogously for $\phi_{L}(\overrightarrow{\mathrm{r}})$. Here $L$ is short for $t$ lu and $U_{q m ; u}$ is an arbitrary unitary matrix of dimension $(2 l+1) h_{t}$. The bracket $\langle||\rangle$ now indicates the sum of integrals in all spheres of the cell and, consequently, $\left\langle\Phi_{L} \cdot \mid \Phi_{L}\right\rangle$ and $\left\langle\Phi_{L} \cdot|H| \Phi_{L}\right\rangle$ vanish when $L^{\prime} \neq L$. The potential parameters only depend on $t$ and $l$ and $t l$ should therefore be substituted for $l$ and $q m$ for $m$. The $t l$ canonical bands $\delta_{t l i}^{\overrightarrow{\mathbf{k}}}$ are the $(2 l+1) h_{t}$ eigenvalues of $\delta_{t l q^{\prime} m^{\prime} ; t l q m}^{\vec{k}}$.

Self-consistent calculations are almost imperative for compounds and for this purpose the ASA is extremely efficient. The spherically symmetrized density of band electrons in a sphere of type $t$ may be expressed as

$$
\begin{aligned}
4 \pi \rho_{t}(r) & =\int d \hat{r} \sum_{j, \overrightarrow{\mathrm{k}}}^{o c c}\left|\Psi_{j}^{\overrightarrow{\mathbf{k}}}(\overrightarrow{\mathrm{r}})\right|^{2} \\
& =\sum_{l} \int_{F}^{E_{F}} N_{t l}(E) \phi_{t l}^{2}(E, r) d E,
\end{aligned}
$$

where the origin is taken at one of the $\vec{Q}_{t q}$ 's and where, upon Taylor expansion of $\phi_{t l}(E, r)$, the integral over energy reduces to a sum involving the energy moments. The number of states in a sphere of type $t$, with angular momentum $l$, and with energies in the interval $(E \mid E+d E)$ is

$$
N_{t l}(E) d E=\sum_{j} \frac{\Omega}{8 \pi^{3}} \int_{(E \mid E+d E)} d^{3} k \frac{1}{h_{t}} \sum_{u}\left|B_{L j}^{\overrightarrow{\mathbf{k}}}\right|^{2},
$$

where, according to (4.23),

$$
B_{L j}^{\prime \overrightarrow{\mathbf{k}}} \equiv \frac{(2 l+1) \chi_{t l}\left(D_{L}\right)}{\left[l-D_{t l}(E)\right] \phi_{t l}(E)} A_{L j}^{\overrightarrow{\mathrm{k}}}\left(D_{L}\right)
$$

and

$$
B_{L j}^{\overrightarrow{\mathrm{k}}}=B_{L j}^{\overrightarrow{\mathrm{k}}}\left(\sum_{L}\left|B_{L j}^{\prime \overrightarrow{\mathrm{k}}}\right|^{2}\right)^{-1 / 2} .
$$

With this atomic-sphere representation of the charge density, the Coulomb potential may easily be found by solution of the radial Poisson equation in each sphere and by calculation of the Madelung shifts employing the $s$-structure constants.

\section{RELATIVISTIC EFFECTS}

In accurate energy-band calculations relativistic effects can only justifiably be neglected for materials of atomic number less than about twenty. The relativistic shift of the $4 s$ band with respect to the $3 d$ band is $-12 \mathrm{mRy}$ in $\mathrm{Ti}(Z=22)$, and the $3 d$ spinorbit coupling parameter equals 1.5 mRy. ${ }^{24}$ For $\mathrm{Ru}(Z=44)$, the corresponding energies are, respectively, -70 and $10 \mathrm{mRy}$ and, for Os $(Z=76)$, they are -230 and $35 \mathrm{mRy}{ }^{1}$ This separation of the relativistic effects into mass-velocity and Darwin shifts, which may be taken into account merely by changing the potential parameters, and the effect of spin-orbit coupling, which alters the symmetry of the Hamiltonian, is not only possible but it is also extremely convenient, because the spin-orbit coupling parameters $\xi_{l}$ are smaller than the relativistic shifts by typically one order of magnitude. Moreover, only in small regions of $\vec{k}$ space, near certain points of degeneracy, is the spin-orbit splitting as large as $\left(l+\frac{1}{2}\right) \xi_{l}$ and even this energy is considerably smaller than the width of the $l$ band.

We shall then, for the purpose of conceptual simplicity and with little or no loss of accuracy, include the relativistic effects in the LAPW and LMTO methods by formally using the Pauli Hamiltonian $^{25}$

$$
H=-\nabla^{2}+v-c^{-2}\left((E-v)^{2}+v^{\prime} \frac{\partial}{\partial r}\right)+\xi \overrightarrow{\mathbf{s}} \cdot \overrightarrow{\mathrm{l}}
$$

inside the spheres, and treating the spin-orbit coupling term

$$
\xi(\overrightarrow{\mathbf{s}} \cdot \overrightarrow{\mathrm{l}})=c^{-2} \frac{2}{r} \frac{d v}{d r} \frac{1}{2}\left(\begin{array}{cc}
l_{z} & l_{-} \\
l_{+} & -l_{z}
\end{array}\right)
$$

as a perturbation.

\section{A. Relativistic formalism for single sphere}

The entire formalism of the previous sections applies for the Pauli Hamiltonian without the spinorbit coupling term, provided that the radial wave functions, and hence the potential parameters, are obtained from radial equations which include the mass-velocity and Darwin terms $-c^{-2}\left[(E-v)^{2}\right.$ $\left.+v^{\prime}(d / d r)\right]$.

The trial function $\Phi_{l m}(D, \overrightarrow{\mathrm{r}})$ is now (2.3) times one of the spin functions, $\uparrow$ or $\downarrow$, and the spin-orbit coupling matrix, to be added to (2.4), is

$$
\left\langle\Phi_{l^{\prime} m^{\prime}}\left(D^{\prime}\right)|\xi \overrightarrow{\mathrm{S}} \cdot \overrightarrow{\mathrm{l}}| \Phi_{l m}(D)\right\rangle=\xi_{l}\left(D^{\prime}, D\right)\left(l^{\prime} m^{\prime} \mid l m\right) \text {. }
$$

Here,

$$
\left(l^{\prime} m^{\prime} \mid l m\right) \equiv \frac{1}{2} \delta_{l^{\prime} l}\left(\begin{array}{cc}
\delta_{m^{\prime} m} m & \delta_{m^{\prime}(m-1)}[(l+m)(l-m+1)]^{1 / 2} \\
\delta_{m^{\prime}(m+1)}[(l-m)(l+m+1)]^{1 / 2} & \delta_{m^{\prime} m}(-m)
\end{array}\right)
$$


and the dependence of the radial integral $\xi_{l}\left(D^{\prime}, D\right)$ $\equiv\left\langle\Phi_{l}\left(D^{\prime}\right)|\xi| \Phi_{l}(D)\right\rangle$ on the logarithmic derivatives may be accounted for as follows. The energy derivative function at $E_{\nu}$ of a radial wave function which is not normalized to unity in the sphere, e.g., $I(E) \phi(E, r)$, is a linear combination of $\phi_{\nu}(r)$ and $\dot{\phi}_{\nu}(r)$ and it may the refore be written as a constant times one of the functions $\Phi(r)$ in (2.2). If we choose $I(E) \phi(E, r)$ to be the solution which results from integrations of the radial equation when the initial condition at $r=0$ is independent of energy, the corresponding energy derivative function $\Phi\left(D^{I}, r\right)$ vanishes (as $\left.r^{l+2}\right)$ near the nucleus where the relativistic operators are effective. We may then assume that $\xi(r) \Phi\left(D^{I}, r\right)$ vanishes everywhere in the sphere and, from the equation obtained from (2.11) when substituting $l$ by $D_{\nu}$ and $-l-1$ by $D^{I}$, i.e.,

$$
\Phi(D, r)=\left(1-\frac{\omega(D)}{\omega\left(D^{I}\right)}\right) \phi_{\nu}(r)+\frac{\omega(D)}{\omega\left(D^{I}\right)} \Phi\left(D^{I}, r\right)
$$

we obtain

$$
\xi\left(D^{\prime}, D\right)=\left(1-\frac{\omega\left(D^{\prime}\right)}{\omega\left(D^{I}\right)}\right)\left(1-\frac{\omega(D)}{\omega\left(D^{I}\right)}\right) \xi_{\nu}
$$

in terms of the two new potential parameters, $\xi_{\nu}$ $\equiv\left\langle\phi_{\nu}|\xi| \phi_{\nu}\right\rangle$ and $D^{I}$. Since $I(E) \phi(E, r)$ have the normalization usually obtained in numerical integrations of the radial equation, the parameter $D^{I}$ is most easily obtained by differentiation of the amplitude at the sphere and using (2.9), i.e. ,

$$
\frac{1}{D_{\nu}-D^{I}}=\left.S \phi_{\nu} \frac{\partial I \phi}{I \partial E}\right|_{E_{\nu}}
$$

For a given boundary condition $D$, angular quantum number $l$, and radial quantum number $\nu$, the Pauli Hamiltonian then has an $(l+1)$-fold degenerate solution of energy $E(D)+\frac{1}{2} l \xi(D)$ and an $l$-fold degenerate solution of energy $E(D)-\frac{1}{2}(l+1) \xi(D)$, where $E(D)$ is given by $(2.17)$ and where the spin-orbit coupling parameter is

$$
\xi(D)=\frac{\xi(D, D)}{1+\left\langle\dot{\phi}_{\nu}^{2}\right\rangle \omega^{2}(D)} .
$$

The corresponding exact energies $E_{k=-l-1}(D)$ and $E_{k=l}(D)$ of the Dirac Hamiltonian are the inverse functions of the Dirac logarithmic derivative functions $^{5,26}$

$$
D_{k}(E)=S \frac{g_{k}^{\prime}(E)}{g_{k}(E)}=-\kappa-1+S \frac{c f_{k}(E)}{g_{\kappa}(E)},
$$

and, since the radial Dirac equations are computationally quite simple, we shall now indicate how the potential parameters may be obtained from the solutions $f_{k}(E, r)$ and $g_{k}(E, r)$ of these. As usual, $\kappa$ is the combined quantum number for $l, j$, and $s=\frac{1}{2}$. With the normalization

$$
\int_{0}^{s}\left[f_{\kappa}^{2}(E, r)+g_{\kappa}^{2}(E, r)\right] r^{2} d r=1
$$

and provided that the kinetic energy in the outer region of the sphere is much less than $c^{2} \approx(2 \times 137)^{2} \mathrm{Ry}$, the representation in Sec. II of the logarithmic derivative functions is valid when $g(E)$ is substituted for $\phi(E)$, and $\kappa$ is substituted for $l$. With the choice of logarithmic derivative

$$
D_{\nu l}=D_{\nu, k=-l-1}=D_{\nu, k=l},
$$

the potential parameters may then be obtained from the Dirac parameters as

$$
\begin{aligned}
& (2 l+1) E_{\nu l}=(l+1) E_{\nu, k=-l-1}+l E_{\nu, k=l}, \\
& (2 l+1) \phi_{\nu l}^{2}=(l+1) g_{\nu,-l-1}^{2}+l g_{\nu, l}^{2}, \\
& (2 l+1) \phi_{\nu l}^{3} \dot{\phi}_{\nu l}=(l+1) g_{\nu,-l-1}^{3} \dot{g}_{\nu,-l-1}+l g_{\nu, l}^{3} \dot{g}_{\nu, l}, \\
& (2 l+1) \phi_{\nu l}^{5} \ddot{\phi}_{\nu l}=(l+1) g_{\nu,-l-1}^{5} \ddot{g}_{\nu,-l-1}+l g_{\nu, l}^{5} \ddot{g}_{\nu, l}, \\
& \left(l+\frac{1}{2}\right) \xi_{\nu l}=E_{\nu, k=-l-1}-E_{\nu, k=l},
\end{aligned}
$$

and

$$
\omega_{l}\left(D_{l}^{I}\right)=(2 l+1) \xi_{\nu l} \phi_{\nu l}^{2} /\left(g_{\nu, l}^{2}-g_{\nu,-l-1}^{2}\right) .
$$

The approximation of attributing the $D$ dependence of the splitting $E_{k=-l-1}(D)-E_{k=l}(D)$ between the Dirac logarithmic derivative functions to the normalization effect described by $(5.2)-(5.4)$ can be justified by comparing the value $\omega\left(D^{I}\right)$, obtained from (5.5), with the mean value

$$
\left[(l+1) \omega_{-l-1}\left(D_{-l-1}^{I}\right)+l \omega_{l}\left(D_{l}^{I}\right)\right] /(2 l+1)
$$

obtained from (5.3) with $\phi$ substituted by $g$; for the $6 p$ parameter of $\mathrm{Pt}(Z=78)$, both values are $-5.3 \mathrm{Ry}$ and, for the $5 d$ parameter, both values are $-1.58 \mathrm{Ry}$.

\section{B. Spin-orbit coupling in LAPW method}

The energy-independent APW is given by (3.1) times one of the spin functions, $\downarrow$ or $\downarrow$, and the spin-orbit coupling matrix to be added to (3.2) or (3.8) is therefore

$$
\Omega^{-1}\left\langle\psi_{\overrightarrow{\mathrm{K}}^{\prime}}|\xi \overrightarrow{\mathrm{s}} \cdot \overrightarrow{\mathrm{I}}| \psi_{\overrightarrow{\mathrm{K}}}\right\rangle=i\left[\overrightarrow{\mathrm{s}} \cdot\left(\overrightarrow{\mathrm{K}} \times \overrightarrow{\mathrm{K}}^{\prime}\right)\right] \frac{4 \pi S}{\Omega} \sum_{l=1}(2 l+1) P^{\prime}\left(\hat{K}^{\prime} \cdot \hat{K}\right) j\left(K^{\prime} S\right) j(K S) \frac{\xi\left(\tilde{D}^{\prime}, \tilde{D}\right)}{S \Phi^{\prime} \Phi},
$$

where $P^{\prime}$ is the derivative of a Legendre polynomial. With the substitution

$$
(2 l+1) \xi_{l}\left(\tilde{D}^{\prime}, \tilde{D}\right) / S \Phi_{l}^{\prime} \Phi_{l} \rightarrow D_{k=-l-1}(E)-D_{k=l}(E)
$$


the matrix (5.6) equals a term of the ordinary, relativistic APW method. ${ }^{26}$

\section{Spin-orbit coupling in LMTO method}

The Bloch sum of energy-independent MTOs is given by (4.2), or its generalization approximate for several atoms per primitive cell, times one of the spin functions, $\uparrow$ or $\downarrow$. The spin-orbit coupling matrix to be added to the Hamiltonian matrix (4.9) or (4.12), is therefore

$$
\begin{aligned}
\left\langle\chi_{L^{\prime}}^{\overrightarrow{\mathbf{k}}}|\xi \overrightarrow{\mathbf{s}} \cdot \vec{l}| \chi_{L}^{\overrightarrow{\mathbf{k}}}\right\rangle= & \xi_{l}\left(D_{L^{\prime}}, D_{L}\right)\left(L^{\prime} \mid L\right)-\left(\xi_{l^{\prime}}\left(D_{L^{\prime}}, l^{\prime}\right) \sum_{L^{\prime \prime \prime}} \frac{\left(L^{\prime} \mid L^{\prime \prime \prime}\right) T_{L}^{\overrightarrow{\mathbf{k}}} \cdots_{L}}{\omega_{l^{\prime}}\left(D_{L^{\prime \prime \prime}}\right)-\omega_{l^{\prime}}\left(l^{\prime}\right)}+\text { H. c. }\right) \\
& +\sum_{L^{\prime \prime}} \frac{T_{L^{\prime} L^{\prime \prime}}^{\overrightarrow{\mathbf{k}}} \xi_{l^{\prime \prime}}\left(l^{\prime \prime}, l^{\prime \prime}\right)}{\omega_{l^{\prime \prime}}\left(D_{L^{\prime \prime}}\right)-\omega_{l^{\prime \prime}}\left(l^{\prime \prime}\right)} \sum_{L^{\prime \prime \prime}} \frac{\left(L^{\prime \prime} \mid L^{\prime \prime \prime}\right) T_{L}^{\overrightarrow{\mathbf{k}}} \cdots_{L}}{\omega_{l^{\prime \prime}}\left(D_{L^{\prime \prime \prime}}\right)-\omega_{l^{\prime \prime}}\left(l^{\prime \prime}\right)} .
\end{aligned}
$$

In the $t l q m$ representation

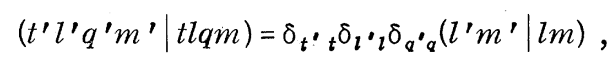

so that the sums on $L^{\prime \prime \prime}$ in the second and third terms of (5.7) reduce to single terms. In the tli representation

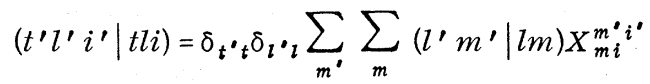

$$
\begin{aligned}
& =\frac{1}{2} \delta_{t^{\prime} t^{\prime} \delta_{l^{\prime} l}}\left(\begin{array}{cc}
\sum_{m} m X_{m i}^{m i^{\prime}} & \sum_{m}[(l+m)(l-m+1)]^{1 / 2} X_{m i}^{m-1 i^{\prime}} \\
\sum_{m}[(l-m)(l+m+1)]^{1 / 2} X_{m i}^{m+1 i^{\prime}} & -\sum_{m} m X_{m i}^{m i^{\prime}}
\end{array}\right),
\end{aligned}
$$

where

$$
X_{m i}^{m^{\prime} i^{\prime}} \equiv \sum_{\alpha} U_{\alpha m^{\prime} ; i^{\prime}}^{\overrightarrow{\vec{k} *}} U_{\alpha m ; i}^{\overrightarrow{\mathbf{k}}}
$$

so that the sums on $L^{\prime \prime \prime}$ are sums over the canonical band index $i$.

The common approximation of retaining the spinorbit coupling in the one-center integral only, amounts to neglecting the variation of the coupling parameter $\xi$ across the band, i.e., its $D$ dependence. This may be seen from (5.7) when choosing $D_{L}=-l-1$, in which case the first term of (5.7) is the true one-center integral. If, on the other hand, we choose $D_{L}=D_{t l i}^{\overrightarrow{\mathbf{k}}}$ and use the $t l i$ representation, it is not a bad approximation to neglect in (5.7) all other terms but the first.

\section{CONCLUSION}

In this paper we have presented the details of the linear-APW and -MTO formalisms and investigated their respective merits. Of the two methods, the LAPW method is the most generally applicable, its accuracy is arbitrary in the sense that $E_{\nu}$ is arbitrary, and it may be used at high energies and for open structures because the plane-wave set is overcomplete in the interstitial region so that non-MT contributions to the potential may be included.

Since the plane-wave part of the energy-independent APW is continued smoothly inside the spheres, the non-MT contributions enter primarily through their Fourier components. When $d$ bands are present, the angular behavior in the interstitial region cannot be represented by less than 25-50 plane waves per atom and, if applicable, the LMTO method is far more efficient. In this case, only the nine $s, p$, and $d$ MTOs are needed per atom but, since the method has additional errors, proportional to $\Omega_{I}^{2}\left(E-V_{\mathrm{mtz}}\right)^{2}$, it is only useful for closely packed structures and low energies. This method is computationally very fast, and it may be simplified through the atomic-sphere approximation and further, through the neglect of hybridization, in which case there are no matrix diagonalizations to be performed; the energy bands are just the canonical bands scaled nonuniformly by the potential parameters. A theory of the electronic structure of hcp transition metals, based on the LMTO method at its various levels of simplicity, is presented in the following paper.

\section{ACKNOWLEDGMENTS}

I wish to express my gratitude to Professor A. R. Mackintosh for his interest, help and encouragement at all stages of the present work. Dr. O. Jepsen made the linear-MTO method work in practice, and performed the computations illustrated in Figs. 5 and 6 . I have benefitted greatly from many fruitful discussions with him. Useful comments from Dr. D. G. Pettifor, Dr. N. Egede Christensen, Dr. H. Skriver, and Dr. G. Arbman are also gratefully acknowledged. 
*Work supported in part by the AEK Research Establishment, Ris $\phi$.

${ }^{1} \mathrm{O}$. Jepsen, O. K. Andersen, and A. R. Mackintosh, following paper, Phys. Rev. B 12, xxxx (1975).

${ }^{2} \mathrm{P}$. Hohenberg and W. Kohn, Phys. Rev. 136, B864 (1964); L. Hedin and B. I. Lundqvist, J. Phys.C 4 , 2064 (1971); U。 von Barth and L. Hedin, ibid. 5, 1629 (1972); O. Gunnarsson, J. Phys. F (to be published); J. C. Slater, Quantum Theory of Molecules and Solids (McGraw-Hill, New York, 1974), Vol. IV.

${ }^{3}$ F。 W。 Averill, Phys。 Rev. B 4, 3315 (1971); T。 M。 Hattox, J。 B。 Conklin, $\mathrm{Jr}_{\circ}, \mathrm{J}_{\circ} \mathrm{C}$. Slater, and $\mathrm{S}$. B。 Trickey, J。 Phys. Chem. Solids 34, 1627 (1973); G。 Arbman and U. von Barth, J. Phys. F 5, 1155 (1975). V. L. Moruzzi, A. R. Williams, and J. F. Janak, Phys. Rev. B 9, 3316 (1974); J. F. Janak, A. R.Williams, and W. L. Moruzzi, ibid. 6, 4367 (1972); 11 , 1522 (1975). Contrary to the conclusion reached by Janak et al. from their nonrelativistic calculations, we believe that the first-principles potential of Hedin and Lundqvist (Ref.2) describes the Fermi surface of $\mathrm{Cu}$ exceedingly well. The reason is that the Dirac potential parameters (Sec. V) obtained from Chodorow's potential show that the relativistic $s-d$ band shift is approximately $-20 \mathrm{mRy}$, which is three times larger than estimated by Janak et al.

${ }^{4} \mathrm{~J}$. Madsen, O. Jepsen, and O. K. Andersen (unpublished).

${ }^{5}$ See, for instance, T. L. Loucks, Augmented Plane Wave Method (Benjamin, New York, 1967); J. O. Dimmock, Solid State Physics (Academic, New York, 1971), Vol。 26.

${ }^{6}$ See, for instance, B. Segall and F. S. Ham, Methods in Computational Physics (Academic, New York, 1968), Vol. 8, Chap. 7 .

7J. M. Ziman, Proc. Phys. Soc. Lond. 86, 337 (1965); J. Hubbard, ibid. 92, 921 (1967); V. Heine, Phys. Rev. 153,673 (1967). For a review, see, N. W. Dalton, Computational Methods in Band Theory (Plenum, New York, 1971).

${ }^{8}$ D. G. Pettifor, J. Phys. C $\underline{5}, 97$ (1972).
${ }^{9}$ See, for instance O. K. Andersen, Phys. Rev。Lett. 27, 1211 (1971).

${ }^{10}$ P. M. Marcus, Int. J. Quantum. Chem. Suppl. 1, 567 (1967).

${ }^{11} \mathrm{O} . \mathrm{K}$. Andersen, in Winter College on Electrons in Crystalline Solids 1972 (IAEA, Vienna, to be published); In Mont Tremblant International Summer School 1973 (unpublished).

${ }^{12} \mathrm{O}$. K. Andersen and R. G. Wooley, Mol. Phys. 26 , 905 (1973).

${ }^{13} \mathrm{~J}$. H. Wilkinson, The Algebraic Eigenvalue Problem (Oxford U. P., London, 1965); R. S. Martin and J. H. Wilkinson, Numer. Math. 12, 377 (1968).

${ }^{14} \mathrm{O}$. K. Andersen, in Computational Methods in Band Theory (Plenum, New York, 1971); O. K. Andersen and R. V. Kasowski, Phys. Rev. B 4, 1064 (1971).

${ }^{15} \mathrm{~J}$. Bardeen, J. Chem. Phys. $\underline{6}, 367$ (1938); R. A. Silverman, Phys. Rev. 85, 227 (1952); H. Brooks, Nuovo Cimento Suppl. 7, 165 (1958). See also, J. Callaway, Energy Band Theory (Academic, New York, 1964).

${ }^{16} \mathrm{O}$. Jepsen and O. K. Andersen (unpublished).

${ }^{17}$ O. K. Andersen, Solid State Commun. 13, 133 (1973).

${ }^{18}$ D. D. Koelling and G. O. Arbman, J. Phys. F (to be published).

${ }^{19} \mathrm{O}$. Jepsen and O. K. Andersen (unpublished).

${ }^{20} \mathrm{~T}$. Jarlborg and G. O. Arbman (unpublished).

${ }^{21} \mathrm{E}$. U. Condon and G. H. Shortley, The Theory of Atomic Spectra (Cambridge U. P., Cambridge, England, 1951).

${ }^{22}$ M. M. Saffren and J. C. Slater, Phys. Rev. 92, 1126 (1953).

${ }^{23}$ For a review see L. F. Mattheiss, J. H. Wood, and A. C. Switendick, in Methods in Computational Physics (Academic, New York, 1968), Vol. 8, and references contained therein.

${ }^{24}$ O. Jepsen, Phys. Rev. B 12, xxxx (1975).

${ }^{25}$ M. E. Rose, Relativistic Electron Theory (Wiley, New York, 1961).

${ }^{26}$ O. K. Andersen, Phys. Rev. B $\underline{2}, 883$ (1970). 